Provided for non-commercial research and education use. Not for reproduction, distribution or commercial use.

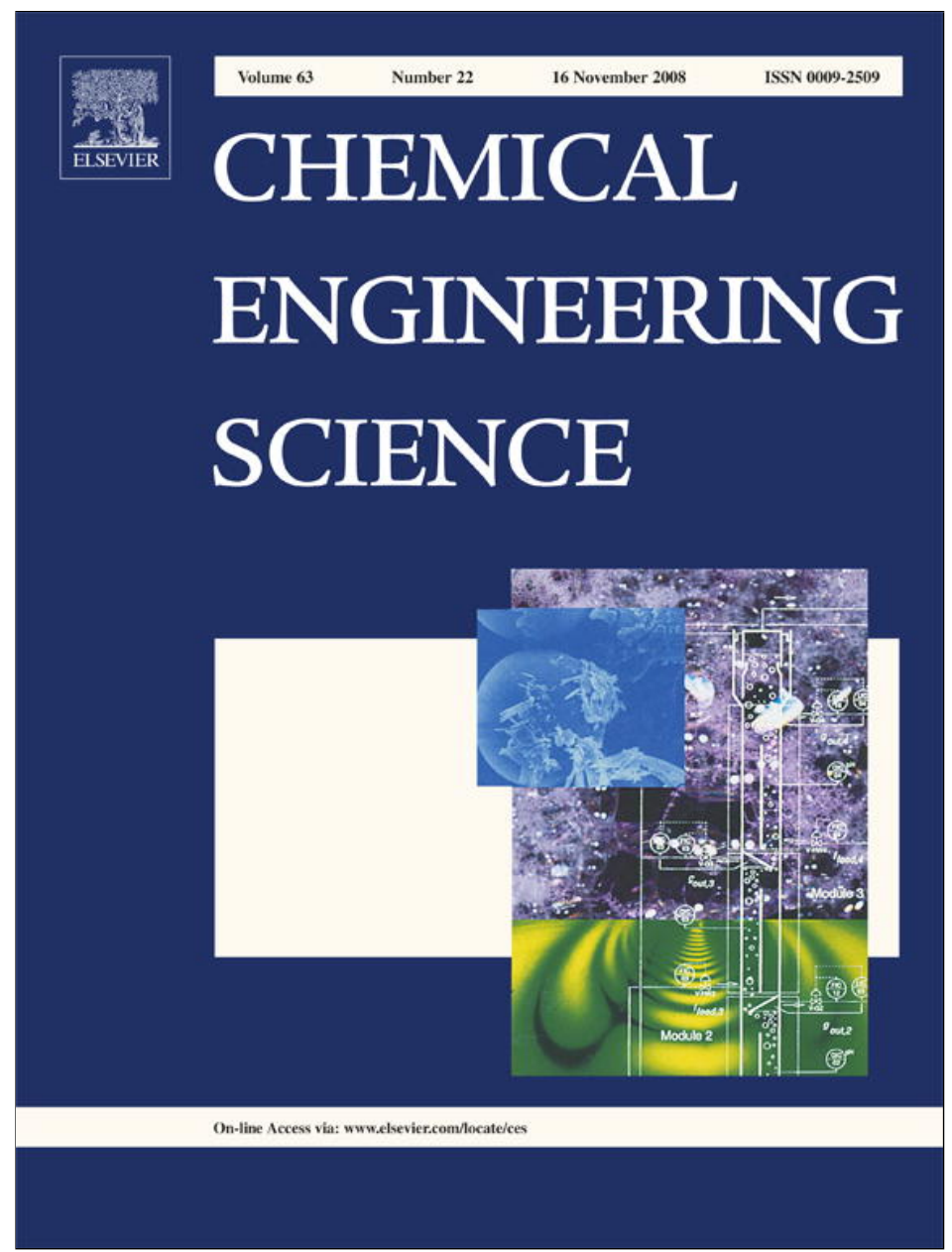

This article appeared in a journal published by Elsevier. The attached copy is furnished to the author for internal non-commercial research and education use, including for instruction at the authors institution and sharing with colleagues.

Other uses, including reproduction and distribution, or selling or licensing copies, or posting to personal, institutional or third party websites are prohibited.

In most cases authors are permitted to post their version of the article (e.g. in Word or Tex form) to their personal website or institutional repository. Authors requiring further information regarding Elsevier's archiving and manuscript policies are encouraged to visit:

http://www.elsevier.com/copyright 


\title{
A two-tier architecture for networked process control
}

\author{
Jinfeng Liu ${ }^{a}$, David Muñoz de la Peña ${ }^{b}$, Benjamin J. Ohran ${ }^{a}$, Panagiotis D. Christofides ${ }^{\mathrm{a}, \mathrm{c}, *}$, James F. Davis ${ }^{\mathrm{a}}$ \\ ${ }^{a}$ Department of Chemical and Biomolecular Engineering, University of California, Los Angeles, CA 90095-1592, USA \\ ${ }^{\mathrm{b}}$ Departmento de Ingeniería de Sistemas y Automática, Universidad de Sevilla, Camino de los Descubrimientos S/N, 41092 Sevilla, Spain \\ 'Department of Electrical Engineering, University of California, Los Angeles, CA 90095-1592, USA
}

\section{A R T I C L E I N F O}

\section{Article history:}

Received 16 May 2008

Received in revised form 23 July 2008

Accepted 28 July 2008

Available online 31 July 2008

\section{Keywords:}

Nonlinear systems

Networked control systems

Model predictive control

Time-varying measurement delays

\begin{abstract}
A B S T R A C T
Traditionally, process control systems utilize dedicated, point-to-point wired communication links using a small number of sensors and actuators to regulate appropriate process variables at desired values. While this paradigm to process control has been successful, chemical plant operation could substantially benefit from an efficient integration of the existing, point-to-point control networks (wired connections from each actuator/sensor to the control system using dedicated local area networks) with additional networked (wired or wireless) actuator/sensor devices. However, augmenting existing control networks with real-time wired/wireless sensor and actuator networks challenges many of the assumptions made in the development of traditional process control methods dealing with dynamical systems linked through ideal channels with flawless, continuous communication. In the context of control systems which utilize networked sensors and actuators, key issues that need to be carefully handled at the control system design level include data losses due to field interference and time delays due to network traffic. Motivated by the above technological advances and the lack of methods to design control systems that utilize hybrid communication networks, in the present work, we present a novel two-tier control architecture for networked process control problems that involve nonlinear processes and heterogeneous measurements consisting of continuous measurements and asynchronous, delayed measurements. This class of control problems arises naturally when nonlinear processes are controlled via control systems based on hybrid communication networks (i.e., point-to-point wired links integrated with networked wired/wireless communication) or utilizing multiple heterogeneous measurements (e.g., temperature measurements which can be taken to be continuous and species concentration measurements which are fed to the control system at asynchronous time instants and frequently involve delays). While point-to-point wired links are very reliable, the presence of a shared communication network in the closed-loop system introduces additional delays and data losses and these issues should be handled at the controller design level. In the two-tier control architecture presented in this work, a lower-tier control system, which relies on point-topoint communication and continuous measurements, is first designed to stabilize the closed-loop system, and an upper-tier networked control system is subsequently designed, using Lyapunov-based model predictive control theory, to profit from both the continuous and the asynchronous, delayed measurements as well as from additional networked control actuators to improve the closed-loop system performance. The proposed two-tier control architecture preserves the stability properties of the lower-tier controller while improving the closed-loop performance. The applicability and effectiveness of the proposed control method is demonstrated using two chemical process examples.
\end{abstract}

(c) 2008 Elsevier Ltd. All rights reserved.

\section{Introduction}

The chemical industry is a key economic sector in the US and globally, and is involved with the conversion of raw materials, through

\footnotetext{
* Corresponding author at: Department of Electrical Engineering, University of California, Los Angeles, CA 90095-1592, USA. Tel.: +1310825 2046;

fax: +13102064107 .

E-mail address: pdc@seas.ucla.edu (P.D. Christofides).
}

a series of chemical processing steps, to valued products. While the range of valuable assets in a chemical plant is large, nearly all the economic value in terms of operating profit is a direct result of plant operations. This realization has motivated extensive research, over the last 40 years, on the development of advanced operation and control strategies to achieve economically optimal plant operation by regulating process variables at appropriate values. With respect to process control, control systems traditionally utilize dedicated, point-to-point wired communication links using a small number of sensors and actuators to regulate appropriate process variables at 
desired values. While this paradigm to process control has been successful, chemical plant operation could substantially benefit from an efficient integration of the existing, point-to-point control networks (wired connections from each actuator/sensor to the control system using dedicated local area networks) with additional networked (wired or wireless) actuator/sensor devices (Ydstie, 2002; Davis, 2007; Neumann, 2007; Christofides et al., 2007). Such an aug mentation in sensor information and network-based availability of wired and wireless data is now well underway in the process industries and clearly has the potential to be transformative in the sense of dramatically improving the ability of the single-process and plant-wide model-based control systems to optimize process and plant performance. Hybrid communication networks allow for easy modification of the control strategy by rerouting signals, having redundant systems that can be activated automatically when component failure occurs, and in general, they allow having a highlevel supervisory control over the entire plant (Christofides and El-Farra, 2005). However, augmenting existing control networks with real-time wired/wireless sensor and actuator networks challenges many of the assumptions made in the development of traditional process control methods dealing with dynamical systems linked through ideal channels with flawless, continuous communication. In the context of hybrid communication networks which utilize networked sensors and actuators, key issues that need to be carefully handled at the control system design level include data losses due to field interference and time delays due to network traffic.

Motivated by the need to develop control architectures that utilize hybrid communication networks, we recently introduced a two-tier control architecture for nonlinear process systems with both continuous and asynchronous measurements (Liu et al., submitted for publication). This class of systems arises in the context of process control systems utilizing hybrid communication networks consisting of point-to-point wired links integrated with wireless actuator/sensor networks. Assuming that there exists a lower-tier control system which relies on point-to-point communication and continuous measurements to stabilize the closed-loop system, we proposed to use Lyapunov-based model predictive control (LMPC) theory (Mhaskar et al., 2005, 2006; Muñoz de la Peña and Christofides, 2008) to design an upper-tier networked control system which profits from both the continuous and the asynchronous measurements as well as from additional networked control actuators. LMPC is based on the concept of uniting model predictive control with control Lyapunov functions as a way of guaranteeing closed-loop stability. The main idea is to formulate appropriate constraints in the predictive controller's optimization problem based on an existing Lyapunov-based controller, in such a way that the MPC inherits the robustness and stability properties of the Lyapunov-based controller. LMPC schemes allow for an explicit characterization of the stability region and lead to reduced complexity optimization problems. We established in Liu et al. (submitted for publication) that the proposed two-tier control system architecture preserves the stability properties of the lower-tier controller while improving the closed-loop performance. However, the developed two-tier control architecture (Liu et al., submitted for publication) does not account for the effect of time-varying network delays as well as measurement sensor delays, which are particularly important during the measurement of species concentrations and particle size distributions in process control applications. Thus, when time-varying measurement delays are present in the closedloop system, the developed two-tier control architecture (Liu et al., submitted for publication) is not guaranteed to maintain the desired closed-loop stability and performance properties.

Within process control, other important recent work on the subject of networked process control includes the development of a quasi-decentralized control framework for multi-unit plants that achieves the desired closed-loop objectives with minimal cross communication between the plant units under state (Sun and El-Farra, 2008) feedback control. In this work, the key ideas are to embed in the local control system of each unit a set of dynamic models that provide an approximation of the interactions between a given unit and its neighbors in the plant when measurements are not transmitted through the plant-wide network and to update the state of each model using measurements from the corresponding unit when communication is re-established. Using a switched system formulation, the maximum allowable update period between the sensors of each unit and the local controllers of its neighbors has been explicitly characterized. In addition to these works, fault diagnosis and faulttolerant control methods that account for network-induced measurement errors have been developed in Ghantasala and El-Farra (2008). In terms of other research work pertaining to the control problem studied in this paper, we note that most of the available results on MPC of systems with delays deal with linear systems (e.g., Jeong and Park, 2005; Liu et al., 2007). Furthermore, the importance of time delays in the context of networked control systems has motivated significant research efforts in modeling such delays and designing control systems to deal with them, primarily in the context of linear systems (e.g., Lian et al., 2003; Montestruque and Antsaklis, 2004; Wang et al., 2005; Zhang et al., 2005; Witrant et al., 2007; Gao et al., 2008). Finally, we recently developed an LMPC technique for nonlinear systems with time-varying measurement delays (Liu et al., in press).

In this work, we continue on our recent efforts (Liu et al., submitted for publication) on the development of two-tier control architectures for networked process control problems. Specifically, we focus on networked process control problems that involve nonlinear processes, hybrid communication networks subjected to data losses and time delays, and heterogeneous measurements consisting of continuous measurements (e.g., temperature measurements) and asynchronous, delayed measurements (e.g., species concentration measurements which are fed to the control system at asynchronous time instants and frequently involve delays). We propose a two-tier control architecture which consists of: (a) a lower-tier control system, which relies on point-to-point communication and continuous measurements, to stabilize the closed-loop system, and (b) an uppertier networked control system, designed using LMPC, that profits from both the continuous and the asynchronous, delayed measurements as well as from additional networked control actuators to improve the closed-loop system performance. The applicability and effectiveness of the proposed control architecture is demonstrated using two chemical process examples.

\section{Preliminaries}

\subsection{Problem formulation}

In this work, we consider nonlinear process systems described by the following state-space model:

$\dot{x}(t)=f\left(x(t), u_{s}(t), u_{d}(t), w(t)\right)$

$y_{S}(t)=h_{S}(x(t))$

$y_{d}(t)=h_{d}(x(t-d(t)))$

where $x(t) \in R^{n_{x}}$ denotes the vector of state variables, $y_{s}(t) \in R^{n_{y_{s}}}$ denotes continuous and synchronous measurements, $y_{d}(t) \in R^{n_{y_{d}}}$ are sampled, asynchronous measurements subject to time-varying measurement delays, $u_{s}(t) \in R^{n_{u_{S}}}$ and $u_{d}(t) \in R^{n_{u_{d}}}$ are two different sets of possible manipulated inputs, $d(t)$ is the size of the time-varying measurement delay (see Section 2.2 below for precise definition of the measurement/network model) and $w(t) \in R^{n_{w}}$ denotes the vector of disturbance variables (i.e., $w(t)$ may include 
unknown/partially known time-varying process parameters and/or external disturbances). The disturbance vector is assumed to be bounded, i.e., $w(t) \in W$ where

$W:=\left\{w \in R^{n_{w}} \text { s.t. }|w| \leqslant \theta, \theta>0\right\}^{1}$

We assume that $f$ is a locally Lipschitz vector function, $h_{S}$ and $h_{d}$ are sufficiently smooth vector functions, and $f(0,0,0,0)=0, h_{s}(0)=0$ and $h_{d}(0)=0$. This means that the origin is an equilibrium point for the nominal system (system (1) with $w(t) \equiv 0$ for all $t$ ) with $u_{s}=0$ and $u_{d}=0$.

Remark 1. The two sets of inputs include both systems with multiple inputs and systems with a single input divided artificially into two terms; i.e.,

$\dot{x}(t)=\hat{f}(x(t), u(t), w(t))$

with $u(t)=u_{s}(t)+u_{d}(t)$

Remark 2. The set of manipulated inputs $u_{d}(t)$ can be used to model actuators controlled via a shared communication network, and hence, possibly affected by data losses or time-varying delays. The set of manipulated inputs $u_{s}(t)$ can be used to model control actuators which are connected with the control system via dedicated, wired links and are guided by a control system that only uses continuous measurements of the outputs $y_{s}(t)$.

\subsection{Modeling of measurements/network}

System (1) is controlled using both continuous synchronous, $y_{s}$, and asynchronous, delayed measurements, $y_{d}$. This class of systems arises naturally in process control applications, where different process variables have to be measured such as temperature, flow rates, species concentrations or particle size distributions. This model is also of interest in the context of processes controlled through a hybrid communication network in which networked wired/wireless sensors and actuators are used to add redundancy to existing control loops (which use point-to-point wired communication links and continuous measurements) because networked communication is often subject to data losses due to field interference (for example, in wireless communication) and time-varying delays due to network traffic. We assume that $y_{s}$ is available for all $t$, while delayed $y_{d}$ samples are received at an asynchronous rate. We also assume that each $y_{d}$ measurement is time-labeled, so the controller is able to discard non-relevant information. Delays in the computation and implementation of control actions can be readily lumped with the measurement delays and are not treated separately. The time instants at which a new delayed $y_{d}$ sample is received are denoted $t_{k}$, where $\left\{t_{k} \geqslant 0\right\}$ is a random increasing sequence of times. To model the timevarying delay, an auxiliary variable $d_{k}$ is introduced to indicate the delay corresponding to the sample received at time $t_{k}$; that is, at time instant $t_{k}$, the sample $y_{d}\left(t_{k}\right)=h_{d}\left(x\left(t_{k}-d_{k}\right)\right)$ is received.

In general, if the sequence $\left\{d_{k} \geqslant 0\right\}$ is modeled using a random process, there exists the possibility of arbitrarily large delays. In this case, it is improper to use all the delayed measurements to estimate the current state and decide the control inputs, because when the delays are too large, they may introduce enough errors to destroy the stability of the closed-loop system. In order to study the stability properties in a deterministic framework, in this paper, we only take advantage of delayed measurements such that the delays associated with the measurements are smaller than an upper bound $D$,

${ }^{1}|\cdot|$ denotes Euclidean norm of a vector.

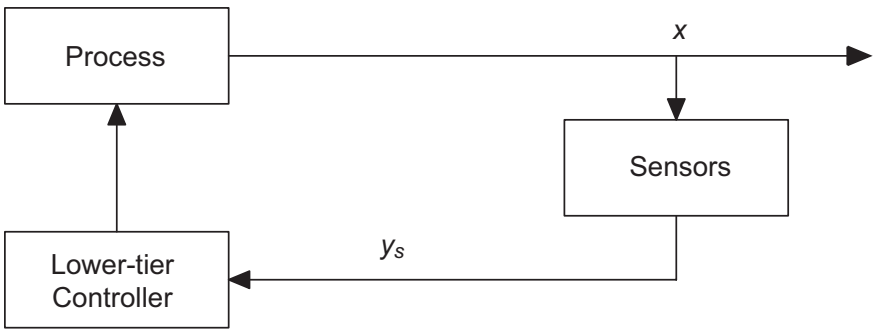

Fig. 1. Lower-tier pre-existing controller with dedicated point-to-point, wired communication links and continuous sensing/actuation.

i.e., $d_{k} \leqslant D, k=0,1 \ldots$. The sequence $\left\{t_{k} \geqslant 0\right\}$ only indicates time instants in which new measurements are available with a corresponding measurement delay lesser than or equal to $D$.

We assume that the measurement of the full state $x$ can be obtained by proper integration of measurements $y_{s}$ and $y_{d}$ at a given time instant. Due to the asynchronous nature of $y_{d}$, the time interval between two consecutive state $x$ measurements is unknown, moreover, due to the time-varying measurement delay of $y_{d}$, the full state $x$ is also subject to time-varying delays. This implies that a controller that is designed to profit from the extra information provided by the asynchronous, delayed measurements $y_{d}$ must take into account that between two consecutive state measurements it has to operate in open-loop and that the received state measurements are delayed so the real state of the system has to be estimated using the nominal model of the system and the available measurement information.

Remark 3. The sequences $\left\{t_{k \geqslant 0}\right\}$ and $\left\{d_{k \geqslant 0}\right\}$ characterize the time needed to obtain a new measurement in the case of asynchronous measurements or the quality of the network link in the case of networked (wired or wireless) communications subject to data losses and time-varying delays. The proposed model is general and can be used to model a wide class of systems subject to asynchronous, delayed measurements.

Remark 4. The sequence $\left\{t_{k} \geqslant 0\right\}$ does not take into account time instants in which a sample that does not provide new information or a sample that involves a delay larger than $D$ is received; that is, the controller discards samples with already known information, or with a delay too long to use this sample to estimate the current state (recall that the measurements are time-labeled).

Remark 5. We have considered that the delayed full state is available asynchronously to simplify the notation. The results can be extended to controllers based on partial state information.

Remark 6. The main idea of the proposed two-tier architecture is to design a controller (see Section 3 below) based not only on the synchronous measurements, but also on the asynchronous measurements to profit from the extra information. This is the case, for example, when extra wireless sensors/actuators are added to an already operating process which is controlled via a control system that uses only continuous, synchronous measurements.

\subsection{Lower-tier controller}

The continuous measurement $y_{s}$ can be used to design a continuous output-feedback controller to stabilize the system. We term the control system based only on the continuous measurements $y_{s}$ as lower-tier controller. This control scheme does not use the delayed measurements $y_{d}$. Fig. 1 shows a schematic of the lower-tier controller which can also be thought of as an already-operating control 
system. To proceed, we assume that there exists an output feedback controller $u_{S}(t)=k_{S}\left(y_{s}(t)\right.$ ) (where $k_{S}\left(y_{s}\right)$ is assumed to be a sufficiently smooth function of $\left.y_{s}\right)$ that renders the origin of the nominal closed-loop system (i.e., $w(t) \equiv 0$ ) asymptotically stable with $u_{d}(t) \equiv 0$. Using converse Lyapunov theorems (see Khalil, 1996), this assumption implies that there exist functions $\alpha_{i}(\cdot), i=1,2,3,4$ of class $\mathscr{K}^{2}$ and a Lyapunov function $V$ for the nominal closed-loop system which is continuous and bounded in $R^{n_{x}}$, that satisfy the following inequalities:

$\alpha_{1}(|x|) \leqslant V(x) \leqslant \alpha_{2}(|x|)$

$\frac{\partial V(x)}{\partial x} f\left(x, k_{S}\left(h_{S}(x)\right), 0,0\right) \leqslant-\alpha_{3}(|x|)$

$\left|\frac{\partial V(x)}{\partial x}\right| \leqslant \alpha_{4}(|x|)$

for all $x \in O \subseteq R^{n_{x}}$ where $O$ is an open neighborhood of the origin. We denote the region $\Omega_{\rho^{3}} \subseteq O$ as the stability region of the closed-loop system under the controller $k_{s}\left(y_{s}\right)$. In the remainder, we will refer to the controller $k_{S}$ as the lower-tier controller. The lower-tier controller based on the output-feedback controller $k_{S}$ is able to stabilize the system, however, it does not profit from the extra information provided by $y_{d}$ and does not utilize $u_{d}(t)$. In what follows, we propose a two-tier control architecture that profits from the extra measurements and control actuators to improve the closed-loop system performance.

Remark 7. The assumption that there exists a lower-tier controller which can stabilize the closed-loop system using only the continuous measurements $y_{S}(t)$ and the inputs $u_{S}(t)$ implies that, in principle, it is not necessary to use the additional information provided by the asynchronous measurements and the extra inputs $u_{d}(t)$ in order to achieve closed-loop stability. However, the main objective of the proposed two-tier control architecture is to profit from this extra information and control effort to improve the closed-loop performance while maintaining the stability properties achieved by the lower-tier controller.

Remark 8. We have considered static lower-tier controllers to simplify the notation. The formulation can be readily extended to dynamic lower-tier controllers as long as they enforce asymptotic stability in the closed-loop system in the sense of (2). In the examples in Sections 4 and 5, proportional-integral (PI) controllers are used as the lower-tier controllers.

Remark 9. The lower tier controller provides some degree of robustness with respect to the uncertainty $w$. Condition (2) and the Lipschitz property of $f$ guarantee that: (a) the closed-loop nominal system under the lower-tier controller is asymptotically stable; (b) the closed-loop system under the lower-tier controller subject to the disturbances is ultimately bounded, provided $\theta$ is sufficiently small, in a region that contains the origin that depends on the size of the uncertainty. These properties are made explicit in Proposition 1 in next section. See Khalil (1996) for more details.

\section{Two-tier architecture}

The main objective of the two-tier control architecture is to improve the performance of the closed-loop system using the information provided by $y_{d}$ while guaranteeing that the stability

${ }^{2}$ Class $\mathscr{K}$ functions are strictly increasing functions of their argument and satisfy $\alpha(0)=0$.

${ }^{3}$ We use $\Omega_{r}$ to denote the set $\Omega_{r}:=\left\{x \in R^{n_{x}} \mid V(x) \leqslant r\right\}$.

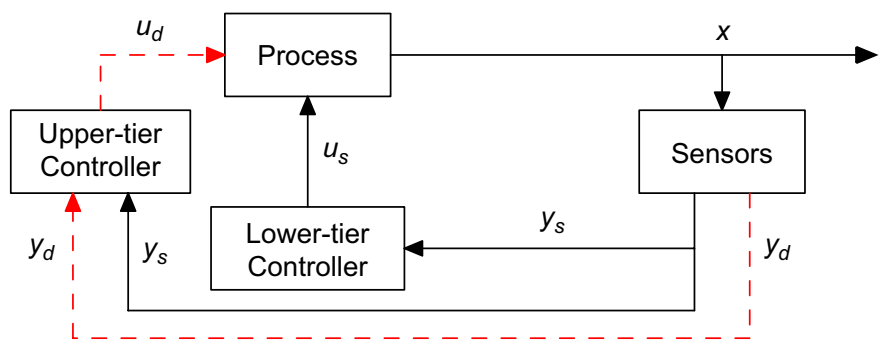

Fig. 2. Two-tier control strategy (solid lines denote dedicated point-to-point, wired communication links and continuous sensing/actuation; dashed lines denote networked (wired/wireless) communication and/or asynchronous sampling/actuation).

properties of the lower-tier controller are maintained. This is done by designing a controller (upper-tier controller) based on the full state measurements obtained by integrating both the continuous and the asynchronous, delayed measurements. In the two-tier control architecture, the upper-tier controller decides the trajectory of $u_{d}(t)$ between successive samples, i.e., for $t \in\left[t_{k}, t_{k+1}\right]$ while the lower-tier controller decides $u_{s}$ using the continuously available measurements. Fig. 2 shows a schematic of the proposed strategy. The upper-tier controller has to take into account time-varying delays and that the time interval between two consecutive samples is unknown. In the following subsections, we present a two-tier architecture based on LMPC theory for the design of the upper-tier controller, which maintains the stability properties of the lowertier controller while improving the closed-loop performance.

Remark 10. Note that since the lower-tier controller has already been designed, this controller views the input $u_{d}$ as a disturbance that has to be rejected if the controller that manipulates $u_{d}$ is not properly designed. Therefore, the design of the upper-tier controller has to take into account the decisions that will be made by the lower-tier controller to maintain closed-loop stability and improve the closed-loop performance.

\subsection{Upper-tier LMPC design}

In order to take advantage of the model of the system and the asynchronous, delayed measurements $y_{d}$, we propose to use LMPC theory to decide $u_{d}$. The main idea of the proposed model predictive controller is the following: at each time instant $t_{k}$ when a new asynchronous measurement $y_{d}\left(t_{k}\right)$ is received, a delayed state measurement $x\left(t_{k}-d_{k}\right)$ is obtained by integrating this measurement with the previously received synchronous measurement $y_{s}\left(t_{k}-d_{k}\right)$. Based on this delayed state measurement $x\left(t_{k}-d_{k}\right)$, the nominal model of the system, the continuous measurements $y_{S}(t)$ and the control inputs applied from $t_{k}-d_{k}$ to $t_{k}$, an estimate of the current state $\tilde{x}\left(t_{k}\right)$ is computed. Note that this implies that the upper-tier controller has to store its past control input trajectory, know the explicit expression and parameters of the lower-tier controller and use the continuous measurements $y_{s}(t)$ to predict the control inputs carried out by the lower-tier controller. The estimated state $\tilde{x}\left(t_{k}\right)$ is then used to obtain the optimal future control input trajectory $u_{d}=u_{d, k}^{*}$ by means of an LMPC optimization problem. This input trajectory is implemented until a new measurement arrives at time $t_{k+1}$. If the time between two consecutive measurements is longer than the prediction horizon, $u_{d}$ is set to zero until a new measurement arrives and the optimal control problem is solved again.

In order to define a finite dimensional optimization problem, $u_{d}$ is constrained to belong to the family of piece-wise constant functions with sampling period $\Delta, S(\Delta)$. To guarantee that the resulting closedloop system is stable, we follow an LMPC approach for the design of 
the upper-tier controller, see Mhaskar et al. $(2005,2006)$ and Muñoz de la Peña and Christofides (2008). LMPC is based on including a contractive constraint that allows to prove practical stability of the closed-loop system using an auxiliary Lyapunov-based controller. In the previous LMPC schemes (Mhaskar et al., 2005, 2006; Muñoz de la Peña and Christofides, 2008), the contractive constraints are defined based on a known Lyapunov-based state feedback controller. In the present work, the contractive constraint of the proposed upper-tier LMPC design is based on the Lyapunov function of the closed-loop system under the lower-tier controller $k_{s}$. Specifically, the upper-tier LMPC optimization problem is defined as follows:

$u_{d, k}^{*}(t)=\arg \min _{u_{d, k} \in S(\Delta)} \int_{t_{k}-d_{k}}^{t_{k}+\tau_{f}} L\left(\tilde{x}(\tau), k_{S}\left(h_{S}(\tilde{x}(\tau))\right), u_{d, k}(\tau)\right) \mathrm{d} \tau$

$\dot{\tilde{x}}(t)=f\left(\tilde{x}(t), k_{s}\left(h_{s}(x(t))\right), u_{d, k}(t), 0\right), \quad \forall t \in\left[t_{k}-d_{k}, t_{k}\right)$

$\dot{\tilde{x}}(t)=f\left(\tilde{x}(t), k_{s}\left(h_{S}(\tilde{x}(t))\right), u_{d, k}(t), 0\right), \quad \forall t \in\left[t_{k}, t_{k}+\tau_{f}\right]$

$\tilde{x}\left(t_{k}-d_{k}\right)=x\left(t_{k}-d_{k}\right)$

$u_{d, k}(t)=u_{d, k-1}^{*}(t), \quad \forall t \in\left[t_{k}-d_{k}, t_{k}\right]$

$\dot{\hat{x}}(t)=f\left(\hat{x}(t), k_{s}\left(h_{S}(\hat{x}(t))\right), 0,0\right), \quad t \in\left[t_{k}, t_{k}+\tau_{f}\right]$

$\hat{x}\left(t_{k}\right)=\tilde{x}\left(t_{k}\right)$

$V(\tilde{x}(t)) \leqslant V(\hat{x}(t)), \quad \forall t \in\left[t_{k}, t_{k}+\tau_{f}\right]$

where $x\left(t_{k}-d_{k}\right)$ is the state obtained integrating both the measurements of $y_{s}\left(t_{k}-d_{k}\right)$ and $y_{d}\left(t_{k}\right)=h_{d}\left(x\left(t_{k}-d_{k}\right)\right), \tilde{x}(t)$ is the predicted trajectory of the two-tier nominal system for the input trajectory computed by the LMPC, $\hat{x}(t)$ is the predicted trajectory of the twotier nominal system for the input trajectory $u_{d}(t) \equiv 0$ for all $t \in$ $\left[t_{k}, t_{k}+\tau_{f}\right], L\left(x, u_{s}, u_{d}\right)$ is a positive definite function of the state and the inputs that define the cost, and $\tau_{f}$ is the prediction horizon. This optimization problem does not depend on the uncertainty and assures that the system in closed-loop with the upper-tier controller maintains the stability properties of the lower-tier controller. The optimal solution to this optimization problem is denoted by $u_{d, k}^{*}(t)$. This signal is defined for all $t>t_{k}$ with $u_{d, k}^{*}(t)=0$ for all $t>t_{k}+\tau_{f}$.

The control inputs of the proposed two-tier control architecture based on the above LMPC are defined as follows:

$u_{S}(t)=k_{S}\left(h_{S}(x(t))\right), \quad \forall t$

$u_{d}(t)=u_{d, k}^{*}(t), \quad \forall t \in\left[t_{k}, t_{k+1}\right)$

where $u_{d, k}^{*}(t)$ is the optimal solution to the LMPC problem at time step $t_{k}$. This implementation technique takes into account that the lower-tier controller uses the continuously available measurements, while the upper-tier controller has to operate in open-loop between consecutive asynchronous, delayed measurements.

Remark 11. Note that by definition, $u_{d, k}^{*}(t)$ is defined for all $t>t_{k}$ with $u_{d, k}^{*}(t)=0$ for all $t>\tau_{f}$. This implies that the upper-tier controller switches off when it has been operating in open-loop for a large time, because in this case, the last received information is no longer useful to improve the performance of the lower-tier controller. Furthermore, we note that the extension of the LMPC scheme of Eq. (3) to the case where the upper-tier controller receives the $y_{s}$ measurements asynchronously subject to delays is conceptually straightforward.

Remark 12. In the proposed LMPC optimization problem both the estimation of $x\left(t_{k}\right)$ from $x\left(t_{k}-d_{k}\right)$ and the evaluation of the future optimal input trajectory in $\left[t_{k}, t_{k+1}\right]$ are carried out at the same time. First, the constraints of the problem guarantee that $\tilde{x}\left(t_{k}\right)$ has been estimated using the nominal model (constraint (3b)) and the real inputs applied to the system (constraint (3e)) from the initial state $x\left(t_{k}-d_{k}\right)$ (constraint (3d)). Once the current state is estimated, the future input trajectory is optimized to minimize the cost function taking into account the lower-tier controller (constraint (3c)) while guaranteeing that the contractive constraint is satisfied (constraint (3h)). This optimization problem has been proposed in order to present a compact controller formulation. It is possible to decouple the observer and the LMPC optimization problem as long as the observer provides an upper bound on the estimation error of $x\left(t_{k}\right)$. For example, a high-gain observer can be used to estimate $x\left(t_{k}\right)$ from the continuous measurements and the applied inputs, and then use this estimated state to define the LMPC optimization problem.

Remark 13. Constraints (3c) and (3h) are a key element of the proposed two-tier control architecture. In general, guaranteeing closed-loop stability of a distributed control scheme is a difficult task because of the interactions between the different controllers and can only be done under certain assumptions (see, for example, Rawlings and Stewart, 2007; Camponogara et al., 2002). Constraint (3c) guarantees that the upper-tier controller takes into account the effect of the lower-tier controller to the applied inputs (recall that the lower-tier controller is designed without taking $u_{d}$ into account). Constraint ( $3 \mathrm{~h}$ ) will be used to guarantee that the value of the Lyapunov function is a decreasing sequence of time with a lower bound.

\subsection{Two-tier controller stability}

Using asynchronous and delayed measurements without proper care in the control system may deteriorate the stability of the closedloop system. In order to maintain the closed-loop system stability properties enforced by the lower-tier controller and improve the performance of the closed-loop system, we propose to follow a Lyapunov-based approach. The main idea, is to compute the input $u_{d}$ applied to the system in a way such that it is guaranteed that in the closed-loop system the value of the Lyapunov function at time instants $t_{k}$; that is, $V\left(x\left(t_{k}\right)\right)$, is a decreasing sequence of values with a lower bound. Following Lyapunov-like arguments, this property guarantees practical stability of the closed-loop system. This is achieved thanks to the contractive constraint of the LMPC optimization problem (3). This property is presented in Theorem 1 below. To state and prove this theorem, we need to state the following three propositions:

Proposition 1 (cf. Khalil, 1996). Consider system (1) in closed-loop with the lower-tier controller. Taking into account (2), there exists a $\mathscr{K} \mathscr{L}^{4}$ function $\beta$, a $\mathscr{K}$ function $\gamma$ and a constant $\theta_{\max }$ such that if $x(0) \in \Omega_{\rho}$ and $u_{d}(t) \equiv 0$ then

$V(x(t)) \leqslant \beta\left(V\left(x\left(t_{0}\right)\right), t-t_{0}\right)+\gamma\left(\max _{\tau \in\left[t_{0}, t\right]}|w(\tau)|\right)$

for all $w \in W$ with $\theta \leqslant \theta_{\max }$.

This proposition provides us with a bound on the trajectories of the Lyapunov function of the state of the system in closed-loop under the lower-tier controller with $u_{d}(t) \equiv 0$. We will use this bound to prove the stability theorem. The proof of Proposition 1 can be found in Khalil (1996).

${ }^{4}$ Function $\beta(r, s)$ is said to be a class $\mathscr{K} \mathscr{L}$ function if, for each fixed $s, \beta(r, s)$
longs to class $\mathscr{K}$ function with respect to $r$ and, for each fixed $r, \beta(r, s)$ is decreasing with respect to $s$ and $\beta(r, s) \rightarrow 0$ as $s \rightarrow 0$. 
Proposition 2. Consider the following state trajectories:

$\dot{x}_{a}(t)=f\left(x_{a}(t), k_{s}\left(h_{s}\left(x_{a}(t)\right)\right), u_{d}(t), w(t)\right)$

$\dot{x}_{b}(t)=f\left(x_{b}(t), k_{s}\left(h_{s}\left(x_{b}(t)\right)\right), u_{d}(t), 0\right)$

with initial states $x_{a}\left(t_{0}\right)=x_{b}\left(t_{0}\right) \in \Omega$. There exists a class $\mathscr{K}$ function $f_{W}(s)$ such that

$\left|x_{a}(t)-x_{b}(t)\right| \leqslant f_{W}\left(t-t_{0}\right)$

for all $x_{a}(t), x_{b}(t) \in \Omega_{\rho}$ and all $w(t) \in W$.

Proof. Define the error vector as $e(t)=x_{a}(t)-x_{b}(t)$. The time derivative of the error is given by

$\dot{e}(t)=f\left(x_{a}(t), k_{S}\left(h_{S}\left(x_{a}(t)\right)\right), u_{d}(t), 0\right)-f\left(x_{b}(t), k_{s}\left(h_{s}\left(x_{b}(t)\right)\right), u_{d}(t), 0\right)$

By continuity and the local Lipschitz property assumed for the vector field $f\left(x, u_{s}, u_{d}, w\right)$, there exist positive constants $L_{w}, L_{x}$ and $L_{u 1}$ such that

$$
\begin{aligned}
|\dot{e}(t)| \leqslant & L_{w}|w(t)-0|+L_{x}\left|x_{a}(t)-x_{b}(t)\right| \\
& +L_{u 1}\left|k_{S}\left(h_{S}\left(x_{a}(t)\right)\right)-k_{S}\left(h_{S}\left(x_{b}(t)\right)\right)\right|
\end{aligned}
$$

for all $x_{a}(t), x_{b}(t) \in \Omega_{\rho}$ and $w(t) \in W$. By continuity and local Lipschitz property of $k_{s}$ and $h_{s}$, there exists a positive constant $L_{u 2}$ such that

$\left|k_{s}\left(h_{s}\left(x_{a}(t)\right)\right)-k_{S}\left(h_{s}\left(x_{b}(t)\right)\right)\right| \leqslant L_{u 2}\left|x_{a}(t)-x_{b}(t)\right|$

Thus, the following inequality can be obtained from inequality (7):

$$
\begin{aligned}
|\dot{e}(t)| & \leqslant L_{w}|w(t)-0|+L_{x}\left|x_{a}(t)-x_{b}(t)\right|+L_{u 1} L_{u 2}\left|x_{a}(t)-x_{b}(t)\right| \\
& \leqslant L_{w} \theta+\left(L_{x}+L_{u 1} L_{u 2}\right)|e(t)|
\end{aligned}
$$

Integrating $|\dot{e}(t)|$ with initial condition $e\left(t_{0}\right)=0$ (recall that $x_{a}\left(t_{0}\right)=$ $\left.x_{b}\left(t_{0}\right)\right)$, the following bound on the norm of the error vector is obtained:

$|e(t)| \leqslant \frac{L_{w} \theta}{L_{x}^{\prime}}\left(e^{L_{x}^{\prime}\left(t-t_{0}\right)}-1\right)$

where $L_{x}^{\prime}=L_{x}+L_{u 1} L_{u 2}$. This implies that Eq. (6) holds for

$f_{W}(\tau)=\frac{L_{w} \theta}{L_{x}^{\prime}}\left(e^{L_{x}^{\prime} \tau}-1\right)$

The following proposition bounds the difference between the magnitudes of the Lyapunov function of two different states in $\Omega_{\rho}$.

Proposition 3. Consider the Lyapunov function $V(\cdot)$ of system (1). There exists a quadratic function $f_{V}(\cdot)$ such that

$V(x) \leqslant V(\hat{x})+f_{V}(|x-\hat{x}|)$

for all $x, \hat{x} \in \Omega_{\rho}$.

Proof. Because the Lyapunov function $V(x)$ is continuous and bounded on compact sets, we can find a positive constant $M$ such that a Taylor series expansion of $V$ around $\hat{x}$ yields

$V(x) \leqslant V(\hat{x})+\frac{\partial V}{\partial x}|x-\hat{x}|+M|x-\hat{x}|^{2}, \quad \forall x, \hat{x} \in \Omega_{\rho}$

Note that the term $M|x-\hat{x}|^{2}$ bounds the high order terms of the Taylor series of $V(x)$ for all $x, \hat{x} \in \Omega \rho$. Taking into account Eq. (2), the following bound for $V(x)$ is obtained:

$V(x) \leqslant V(\hat{x})+\alpha_{4}\left(\alpha_{1}^{-1}(\rho)\right)|x-\hat{x}|+M|x-\hat{x}|^{2}, \quad \forall x, \hat{x} \in \Omega \rho$

This implies that Eq. (8) holds for $f_{V}(x)=\alpha_{4}\left(\alpha_{1}^{-1}(\rho)\right) x+M x^{2}$.

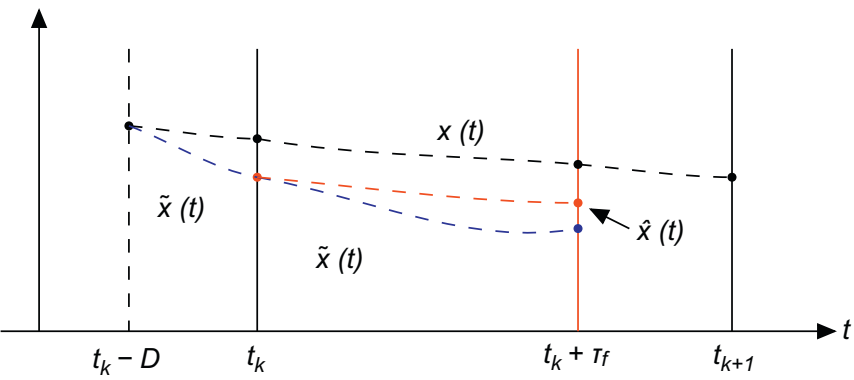

Fig. 3. Possible worst scenario of the delayed measurements received by the upper-tier controller and the corresponding state trajectories defined in problem (3).

We are now in a position to state and prove the main stability result for the proposed two-tier control architecture.

Theorem 1. Consider system (1) in closed-loop with the two-tier control architecture (4). If $x\left(t_{0}\right) \in \Omega_{\rho}, \theta \leqslant \theta_{\max }$, and there exist a concave function $g$ such that

$g(x) \geqslant \beta\left(x+f_{V}\left(f_{W}(D)\right), \tau_{f}\right)$

for all $x \in \Omega \rho$, and a positive constant $c \leqslant \rho$ such that

$c-g(c) \geqslant f_{V}\left(f_{W}\left(D+\tau_{f}\right)\right)$

then $x(t)$ is ultimately bounded in a region that contains the origin.

Proof. In order to prove that system (1) in closed-loop under the two-tier control architecture using the proposed LMPC (3) is ultimately bounded in a region that contains the origin, we will prove that the value of the Lyapunov function at times $\left\{t_{k} \geqslant 0\right\}, V(x)$, is a decreasing sequence of values with a lower bound on its magnitude for the worst possible case from the communications point of view, and hence for all possible sequences of measurement times and delays. The worst possible case from the communications point of view is that the measurements used to evaluate the upper-tier LMPC controller are always received with the maximum delay $D$; that is $d_{k}=D$ for all $k$, and that the upper-tier controller always operates in openloop for a period of time longer than $\tau_{f}$ between consecutive sampling times, that is, $t_{k+1}-t_{k}>\tau_{f}$ for all $k$. If the measurements are received with a smaller delay or more often, the LMPC has more precise information of the state of the system.

Fig. 3 shows the worst scenario for a system of dimension 1 . Solid vertical lines are used to indicate the times at which new measurements are obtained $\left(t_{k}\right.$ and $\left.t_{k+1}\right)$ and when the upper-tier controller switches off at time $t_{k}+\tau_{f}$. The dashed vertical line indicates the time corresponding to the measurement obtained at $t_{k}$ (that is, $t_{k}-D$ ). In this figure, three different state trajectories are shown. The real state trajectory of system (1) (including the uncertainty) is denoted as $x(t)$. The estimated state trajectory from $t_{k}-D$ to $t_{k}$ and the predicted sampled trajectory under the two-tier control architecture computed by the LMPC (3) along the prediction horizon with initial state the estimated state are denoted as $\tilde{x}(t)$. The nominal trajectory under the lower-tier controller $k_{s}$ with $u_{d} \equiv 0$ along the prediction horizon with initial state the estimated state $\tilde{x}\left(t_{k}\right)$ is denoted as $\hat{x}(t)$. The state trajectories $\tilde{x}(t)$ and $\hat{x}(t)$ are obtained using the two-tier nominal model as defined in the LMPC optimization problem (3).

The trajectory $\hat{x}(t)$ corresponds to the nominal system in closedloop with the lower-tier controller with initial state $\tilde{x}\left(t_{k}\right)$. Taking into account Proposition 1 the following inequality holds:

$V(\hat{x}(t)) \leqslant \beta\left(V\left(\tilde{x}\left(t_{k}\right)\right), t-t_{k}\right)$ 
The contractive constraint of the proposed LMPC guarantees that

$V(\tilde{x}(t)) \leqslant V(\hat{x}(t)), \quad \forall t \in\left[t_{k}, t_{k}+\tau_{f}\right]$

Taking into account constraints (3b)-(3d) and that the closed-loop trajectories are defined by the following equation:

$\dot{x}(t)=f\left(x(t), k_{S}\left(h_{s}(x(t))\right), u_{d, k}(t), w(t)\right)$

we can apply Proposition 2 to obtain the following upper bounds on the deviation of $\tilde{x}(t)$ from $x(t)^{5}$ :

$$
\begin{aligned}
& \left|x\left(t_{k}\right)-\tilde{x}\left(t_{k}\right)\right| \leqslant f_{W}(D) \\
& \left|x\left(t_{k}+\tau_{f}\right)-\tilde{x}\left(t_{k}+\tau_{f}\right)\right| \leqslant f_{W}\left(\tau_{f}+D\right)
\end{aligned}
$$

From Proposition 3 and the above inequalities, we obtain the following inequalities:

$$
\begin{aligned}
& V\left(\tilde{x}\left(t_{k}\right)\right) \leqslant V\left(x\left(t_{k}\right)\right)+f_{V}\left(f_{W}(D)\right) \\
& V\left(x\left(t_{k}+\tau_{f}\right)\right) \leqslant V\left(\tilde{x}\left(t_{k}+\tau_{f}\right)\right)+f_{V}\left(f_{W}\left(D+\tau_{f}\right)\right)
\end{aligned}
$$

From inequalities (10)-(12), the following upper bound on $V\left(x\left(t_{k}+\right.\right.$ $\left.\tau_{f}\right)$ ) is obtained:

$$
V\left(x\left(t_{k}+\tau_{f}\right)\right) \leqslant \beta\left(V\left(x\left(t_{k}\right)\right)+f_{V}\left(f_{W}(D)\right), \tau_{f}\right)+f_{V}\left(f_{W}\left(D+\tau_{f}\right)\right)
$$

Taking into account that for all $t>t_{k}+\tau_{f}$ the upper-tier controller is switched off, i.e., $u_{d}(t)=0$, and only the lower-tier controller is in action, the following bound on $V\left(x\left(t_{k+1}\right)\right)$ is obtained from Proposition 1:

$V\left(x\left(t_{k+1}\right)\right) \leqslant \max \left\{V\left(x\left(t_{k}+\tau_{f}\right)\right), \gamma\left(\theta_{\max }\right)\right\}$

for all $w(t) \in W$. Because function $g$ is concave, $z-g(z)$ is an increasing function. If there is a constant $c_{0} \leqslant c \leqslant \rho$ satisfying Eq. (9), then Eq. (9) holds for all $z>c$. Taking into account that $g(z) \geqslant \beta\left(z+f_{V}\left(f_{W}(D)\right), \tau_{f}\right)$ for all $z \leqslant \rho$, the following inequality is obtained:

$z-\beta\left(z+f_{V}\left(f_{W}(D)\right), \tau_{f}\right) \geqslant f_{V}\left(f_{W}\left(D+\tau_{f}\right)\right)$

when $c \leqslant z \leqslant \rho$. From this inequality and inequality (13), we obtain that

$V\left(x\left(t_{k+1}\right)\right) \leqslant \max \left\{V\left(x\left(t_{k}\right)\right), \gamma\left(\theta_{\max }\right)\right\}$

for all $V\left(x\left(t_{k}\right)\right) \geqslant c$. It follows using Lyapunov arguments that

$\limsup _{t \rightarrow \infty} V(x(t)) \leqslant \rho_{c}$

$t \rightarrow \infty$

where

$$
\begin{aligned}
\rho_{c}= & \max \left\{\max _{c} \beta\left(c+f_{V}\left(f_{W}(D)\right), \tau_{f}\right)\right. \\
& \left.+f_{V}\left(f_{W}\left(D+\tau_{f}\right)\right), \gamma\left(\theta_{\max }\right)\right\} .
\end{aligned}
$$

Remark 14. In general the size of the region in which the state is ultimately bounded, depends on the maximum delay $D$ and the prediction horizon $\tau_{f}$. The upper bound on delay $D$ sets the largest size of delay allowed in the measurements used to evaluate the uppertier controller and the prediction horizon $\tau_{f}$ sets the maximum amount of time in which the upper-tier controller will be operating in open-loop.

\footnotetext{
${ }^{5}$ Proposition 2 is used to obtain a bound on the difference between $\tilde{x}$ and $x$ from $t_{k}-d_{k}$ to $t_{k}$ to simplify the notation and the proof. Note that from $t_{k}-d_{k}$ to $t_{k}$, the real trajectory of $u_{\mathrm{s}}$ is applied to evaluate $\tilde{x}$, so tighter bound on the difference between $\tilde{x}$ and $x$ can be obtained. As mentioned before, the estimation of $x\left(t_{k}\right)$ can be done using any observer which provides a bound on the estimation error.
}

Remark 15. Referring to Theorem 1 , the assumption that there exists a concave function $g$ such that $g(x) \geqslant \beta\left(x+f_{V}\left(f_{W}(D)\right), \tau_{f}\right)$ for all $x \in$ $\Omega_{\rho}$ imposes upper bounds on $D$ and $\tau_{f}$ and is made, without any loss of generality, to simplify the proof of Theorem 1 ; i.e., the result of Theorem 1 could still be proved without this assumption but the proof would be more involved. The assumption that there exists a positive constant $c \leqslant \rho$ such that $c-g(c) \geqslant f_{V}\left(f_{W}\left(D+\tau_{f}\right)\right)$ guarantees that the derivative of the Lyapunov function of the state of the closedloop system outside the level set $V(x)=c$ is negative under the twotier control architecture with the upper-tier LMPC (3).

Remark 16. Although the proofs provided are constructive, the constants obtained are conservative. This is the case with most of the results of the type presented in the literature, see for example Nešić et al. (1999) and Tabuada and Wang (2006) for further discussion on this issue. In practice, the maximum time that the system can operate in open-loop is better estimated through closed-loop simulation. The various inequalities proved in this theorem are more useful as guidelines on the interaction between the various parameters that define the system and the controller and may be used as guidelines to design the controller.

\section{Application to a chemical reactor}

\subsection{Process description and modeling}

Consider a well mixed, non-isothermal continuous stirred tank reactor (CSTR) where three parallel irreversible elementary exothermic reactions take place of the form $A \rightarrow B, A \rightarrow C$ and $A \rightarrow D$. Product $B$ is the desired product and $C$ and $D$ are byproducts. The feed to the reactor consists of $A$ at temperature $T_{A 0}$ and concentration $C_{A 0}$ and flow rate $F+\Delta F$, where $\Delta F$ is a time-varying uncertainty. Due to the non-isothermal nature of the reactor, a jacket is used to remove/provide heat from/to the reactor. Using first principles and standard modeling assumptions, the following mathematical model of the process is obtained:

$$
\begin{aligned}
& \frac{\mathrm{d} T}{\mathrm{~d} t}=\frac{F+\Delta F}{V_{r}}\left(T_{A 0}-T\right)-\sum_{i=1}^{3} \frac{\Delta H_{i}}{\sigma c_{p}} k_{i 0} \mathrm{e}^{-E_{i} / R T} C_{A}+\frac{Q}{\sigma C_{p} V_{r}} \\
& \frac{\mathrm{d} C_{A}}{\mathrm{~d} t}=\frac{F+\Delta F}{V_{r}}\left(C_{A 0}-C_{A}+\Delta C_{A 0}\right)+\sum_{i=1}^{3} k_{i 0} \mathrm{e}^{-E_{i} / R T} C_{A}
\end{aligned}
$$

where $C_{A}$ denotes the concentration of the reactant $A, T$ denotes the temperature of the reactor, $V_{r}$ denotes the volume of the reactor, $\Delta H_{i}, k_{i 0}$ and $E_{i}, i=1,2,3$ denote the enthalpies, pre-exponential constants and activation energies of the three reactions, respectively, and $c_{p}$ and $\sigma$ denote the heat capacity and the density of the fluid in the reactor, respectively. The inputs to the system are the rate of heat input/removal $Q$ and the change of the inlet reactant $A$ concentration $\Delta C_{A 0}$. The values of the process parameters are presented in Table 1.

System (14) has three steady-states (two locally asymptotically stable and one unstable). The control objective is to stabilize the system at the open-loop unstable steady-state $T_{S}=388 \mathrm{~K}, C_{A S}=3.59 \mathrm{~mol} / \mathrm{l}$. The flow rate uncertainty is bounded by $|\Delta F| \leqslant 4 \mathrm{~m}^{3} / \mathrm{h}$.

The process model (14) belongs to the class of nonlinear systems described by system (1) where $x^{\mathrm{T}}=\left[\begin{array}{ll}x_{1} & x_{2}\end{array}\right]=\left[T-T_{S} C_{A}-C_{A s}\right]$ is the state, $u_{s}=Q$ and $u_{d}=\Delta C_{A 0}$ are the manipulated inputs, $\theta=\Delta F$ is a time varying bounded disturbance, $y_{S}=x_{1}=T-T_{S}$ is obtained from the continuous temperature measurements $T$ and $y_{d}=x_{2}=C_{A}-C_{A s}$ is obtained at time instants $\left\{t_{k} \geqslant 0\right\}$ from the asynchronously sampled concentration measurement $C_{A}$ subject to time-varying measurement delays. We also have a lower bound $T_{\min }=0.15 \mathrm{~h}$ on the time interval between two consecutive concentration measurements and an upper bound $D$ on the size of the delay; both will be 
Table 1

Process parameters

\begin{tabular}{ll}
\hline$F$ & $4.998 \mathrm{~m}^{3} / \mathrm{h}$ \\
$V_{r}$ & $1 \mathrm{~m}^{3}$ \\
$R$ & $8.314 \mathrm{KJ} / \mathrm{kmol} \mathrm{K}$ \\
$T_{A 0}$ & $300 \mathrm{~K}$ \\
$C_{A 0 s}$ & $4 \mathrm{kmol} / \mathrm{m}^{3}$ \\
$\Delta H_{1}$ & $-5.0 \times 10^{4} \mathrm{KJ} / \mathrm{kmol}$ \\
$\Delta H_{2}$ & $-5.2 \times 10^{4} \mathrm{KJ} / \mathrm{kmol}$ \\
$\Delta H_{3}$ & $-5.4 \times 10^{4} \mathrm{KJ} / \mathrm{kmol}$ \\
$k_{10}$ & $3 \times 10^{6} \mathrm{~h}^{-1}$ \\
$k_{20}$ & $3 \times 10^{5} \mathrm{~h}^{-1}$ \\
$k_{30}$ & $3 \times 10^{5} \mathrm{~h}^{-1}$ \\
$E_{1}$ & $5 \times 10^{4} \mathrm{KJ} / \mathrm{kmol}$ \\
$E_{2}$ & $7.53 \times 10^{4} \mathrm{KJ} / \mathrm{kmol}$ \\
$E_{3}$ & $7.53 \times 10^{4} \mathrm{KJ} / \mathrm{kmol}$ \\
$\sigma$ & $1000 \mathrm{~kg} / \mathrm{m}^{3}$ \\
$c_{p}$ & $0.231 \mathrm{KJ} / \mathrm{kg} \mathrm{K}$ \\
\hline
\end{tabular}

computed via simulations even though conservative estimates could be computed from the theoretical results.

To model the time sequence $\left\{t_{k} \geqslant 0\right\}$, we use a lower-bounded random Poisson process. The Poisson process is defined by the number of events per unit time $W$. The interval between two consecutive concentration sampling times (events of the Poisson process) is given by $\Delta_{a}=\max \left\{T_{\min },-\ln \chi / W\right\}$, where $\chi$ is a random variable with uniform probability distribution between 0 and 1 . In order to model the delay size sequence $\left\{d_{k} \geqslant 0\right\}$, the size of delay associated with the concentration measurement at $t_{k}$ is modeled by an upperbounded random process given by $d_{k}=\min \{D, \phi L\}$, where $\phi$ is a uniformly distributed variable between 0 and 1 , and $L=t_{k}-t_{k-1}+d_{k-1}$ is the size of the time interval between current time $t_{k}$ and the time corresponding to the last concentration measurement $t_{k-1}-d_{k-1}$. This generation method guarantees that $d_{k} \leqslant D$ for all $k$. We assume that the initial state is known; that is, $d_{0}=0$ and $t_{0}=0$.

\subsection{Lower-tier controller design}

An output feedback controller (lower-tier controller) based on the continuous temperature measurements (i.e., $x_{1}(t)$ ) is first designed to stabilize system (14) using only the rate of heat input $u_{s}=Q$ as the manipulated input. In particular, the following PI control law is used as the lower-tier controller:

$u_{S}(t)=K\left(x_{1}(t)+\frac{1}{T_{i}} \int_{0}^{t} x_{1}(\tau) \mathrm{d} \tau\right)$

where $K$ is the proportional gain and $T_{i}$ is the integral time constant. To compute the parameters of the PI controller, the linearized model $\dot{x}=A x+B u_{s}$ of system (14) around the equilibrium point is obtained. The proportional gain $K$ is chosen to be -8100 . This value guarantees that the origin of $\dot{x}=(A+B K[10]) x$ is asymptotically stable with its eigenvalues being $\lambda_{1}=-1.06 \times 10^{5}$ and $\lambda_{2}=-4.43$. A quadratic Lyapunov function $V(x)=x^{\mathrm{T}} P x$ with

$P=\left[\begin{array}{cc}0.024 & 5.21 \\ 5.21 & 1.13 \times 10^{3}\end{array}\right]$

is obtained by solving an algebraic Lyapunov equation $A_{c}^{\mathrm{T}} P+P A_{C}+$ $Q_{c}=0$ for $P$ with $A_{C}=A+B K[10]$. This Lyapunov function will be used to design the upper-tier LMPC controller. The integral time constant is chosen to be $T_{i}=49.6 \mathrm{~h}$. For simplicity, the Lyapunov function $V(x)$ is determined on the basis of the closed-loop system under only the proportional $(P)$ term of the PI controller; the effect of the integral $(I)$ term is very small for the specific choice of the controller parameters used in the simulations and does not change the local stability property of the equilibrium point of the closed-loop system enforced by the proportional part of the PI controller.
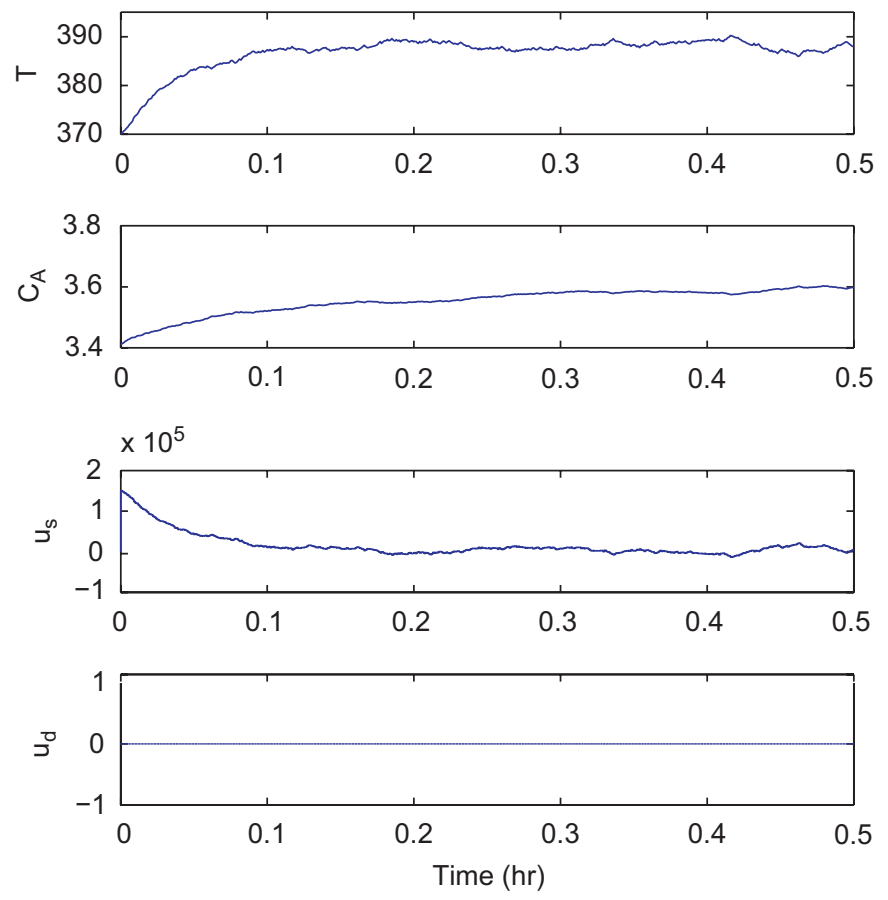

Fig. 4. State and input trajectories of system (14) under lower-tier PI control (15) $\left(u_{d} \equiv 0\right)$.

The state and input trajectories of system (14) starting from $x_{0}=$ [370 3.41 $]^{\mathrm{T}}$ under the PI controller are shown in Fig. 4. From Fig. 4, we see that the PI controller (15) drives the temperature and concentration states of system (14) close to the equilibrium point in about 0.1 and $0.2 \mathrm{~h}$, respectively.

\subsection{Two-tier control architecture design}

We have implemented the proposed two-tier control architecture to improve the performance of the closed-loop system obtained under PI-only control. In this set of simulations, the PI controller (15) with the same parameters is used as the lower-tier controller. Instead of abandoning the less frequent concentration measurements, we take advantage of both the continuous measurements of the temperature, $T$, and the asynchronous, delayed measurements of the concentration, $C_{A}$, together with the nominal model of system (14) to design and implement the upper-tier LMPC. The inlet concentration change $\Delta C_{A 0}$ is the manipulated input for the LMPC.

The LMPC is designed next. The performance index is defined by the following positive definite function:

$L\left(x, u_{s}, u_{d}\right)=x^{\mathrm{T}} Q_{c} x$

where $x$ is the state of the system and $Q_{c}$ is the following weight matrix:

$Q_{C}=\left[\begin{array}{cc}1 & 0 \\ 0 & 10^{4}\end{array}\right]$

The values of the weights in $Q_{c}$ have been chosen to account for the different range of numerical values for each state. The sampling time of the LMPC is $\Delta=0.025 \mathrm{~h}$; the prediction horizon is chosen to be $\tau_{f}=6 \Delta$ so that the prediction captures most of the dynamic evolution of the process; the quadratic control Lyapunov function $V(x)$ is used in the design of the contractive constraint (3h) and the inlet concentration change $\Delta C_{A 0}$ is bounded by $\left|u_{d}\right|=\left|\Delta C_{A 0}\right| \leqslant 1 \mathrm{kmol} / \mathrm{m}^{3}$. 

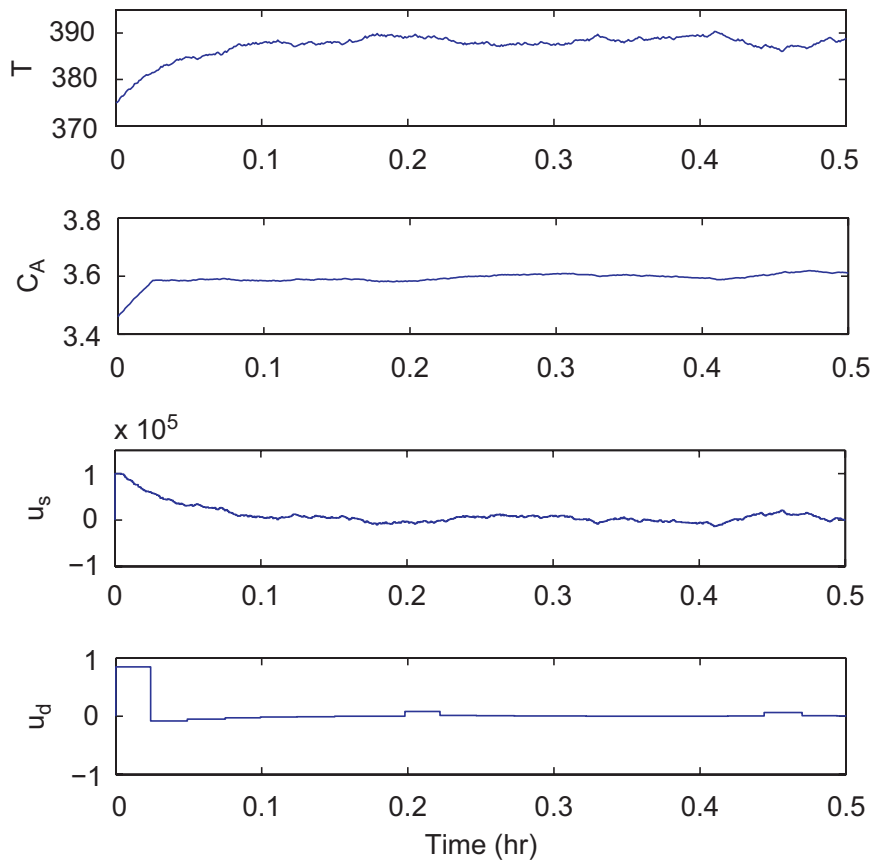

Fig. 5. Worst case state and input trajectories of system (14) under the proposed two-tier control architecture.

For the simulations carried out in this subsection, we pick the delay of each measurement to be $d_{k}=D=0.15 \mathrm{~h}$ for all $k$. These settings correspond to the worst-case effect from a communication point of view. Note that the minimum time interval between two consecutive concentration measurements $T_{\min }$ is fixed by the system dynamics and the prediction horizon is set be equal to the minimum time interval between two consecutive $y_{d}$ measurements, that is $\tau_{f}=T_{\min }$.

The two-tier control architecture is implemented as discussed in the previous section. The lower-tier controller uses the continuous temperature measurements to decide $u_{s}(t)$. When a new measurement of $C_{A}$ is obtained at time instant $t_{k}$ with delay $D$, an estimate of the state of system (14), $x\left(t_{k}-D\right)$, is obtained by integrating the concentration measurement and the previously received continuous measurement of the temperature $T$. Based on the state $x\left(t_{k}-D\right)$, the model of the process and the control actions applied, an estimate of the current state $\tilde{x}\left(t_{k}\right)$ is obtained. Based on this state estimate $\tilde{x}\left(t_{k}\right)$, the LMPC optimization problem (3) is solved and an optimal input trajectory $u_{d, k}^{*}(\tau)$ is obtained. This optimal input trajectory is implemented until a new concentration measurement is obtained at time $t_{k+1}$ (note that $k$ indexes the number of concentration samples received, not a given sampling time). Note that because a PI controller is used in the lower-tier, we need to incorporate the PI controller dynamics in the optimization problem of the proposed LMPC scheme (3).

A simulation of the closed-loop system under the two-tier control architecture (4) with the same initial conditions $x(0)=\left[\begin{array}{ll}370 & 3.41\end{array}\right]^{\mathrm{T}}$ has been carried out. The sampling sequence $\left\{t_{k} \geqslant 0\right\}$ generated with $W=1$ and delay size sequence $\left\{d_{k} \geqslant 0\right\}$ with simulation length of $0.5 \mathrm{~h}$ are the following:

$$
\begin{aligned}
& \left\{t_{k} \geqslant 0\right\}=\left\{\begin{array}{llll}
0 & 0.198 & 0.395 & 0.500
\end{array}\right\} \mathrm{h}
\end{aligned}
$$

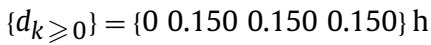

The state and input trajectories of system (14) under the proposed two-tier control architecture are shown in Fig. 5. From Fig. 5, we see that the two-tier control architecture (4) stabilizes the temperature
Table 2

Total performance cost along the closed-loop system trajectories

\begin{tabular}{ccr}
\hline Sim. & Two-tier & PI control \\
\hline 1 & 107.60 & 557.06 \\
2 & 124.98 & 1090.29 \\
3 & 188.53 & 1392.73 \\
4 & 169.06 & 403.82 \\
5 & 143.07 & 376.15 \\
6 & 179.22 & 1330.25 \\
7 & 202.28 & 1252.36 \\
8 & 152.23 & 749.93 \\
9 & 141.84 & 732.20 \\
10 & 157.99 & 1049.38 \\
\hline
\end{tabular}

and concentration of the system at the desired equilibrium point in about 0.1 and $0.05 \mathrm{~h}$, respectively. This implies that the resulting closed-loop system response is faster for this particular simulation. Moreover, the cost associated with the resulting closed-loop trajectories is lower. This result has been validated by extensive simulations.

Remark 17. We considered a performance index $L\left(x, u_{s}, u_{d}\right)$ that penalizes only the closed-loop system state and not the control action because the two-tier control architecture utilizes different manipulated inputs from the lower-tier PI controller and this would complicate the comparison if penalty on the control action is included in the cost. Since the performance index has only penalty on the closed-loop system state, we have included an input constraint on the upper-tier manipulated input, $\Delta C_{A 0}$, to avoid computation of unnecessarily large control actions by the upper-tier controller (i.e., $\left.\left|u_{d}\right| \leqslant 1 \mathrm{kmol} / \mathrm{m}^{3}\right)$.

\subsection{Performance comparison}

We also carried out a set of simulations to compare the proposed two-tier control architecture with the lower-tier PI control system from a performance index point of view. Table 2 presents the total cost computed for 20 different closed-loop simulations under the proposed two-tier control architecture and the PI control scheme. To carry out this comparison, we have computed the total cost of each simulation based on the integral of the performance index defined by $L\left(x, u_{s}, u_{d}\right)$ of Eq. (16) from the initial time to the end of the simulation $t_{f}=0.5 \mathrm{~h}$. For each pair of simulations (one for each control scheme) a different initial state inside the stability region, a different uncertainty trajectory and a different random concentration measurement sequence with random delay size sequence are generated using the methods described in Section 4.1. As it can be seen in Table 2, the proposed two-tier control architecture has a cost lesser than the corresponding total cost under the PI controller in all the closed-loop system simulations.

Remark 18. Note that in this particular example, the improvement in the closed-loop performance is achieved due to the extra control input $u_{d}$ which is guided by the LMPC scheme (3) that uses all available measurements. Since PI controller is used as the lower-tier controller, the extra available asynchronous measurements would not have changed the closed-loop performance achieved by the lowertier controller because the PI controller cannot use the extra measurements. This is also the case for the next example.

\section{Application to a reactor-separator process}

\subsection{Process description and modeling}

The process considered in this example is a three vessel, reactor-separator system consisting of two CSTRs and a flash tank separator (see Fig. 6). A feed stream to the first CSTR $F_{10}$ contains the reactant $A$ which is converted into the desired product $B$. The 


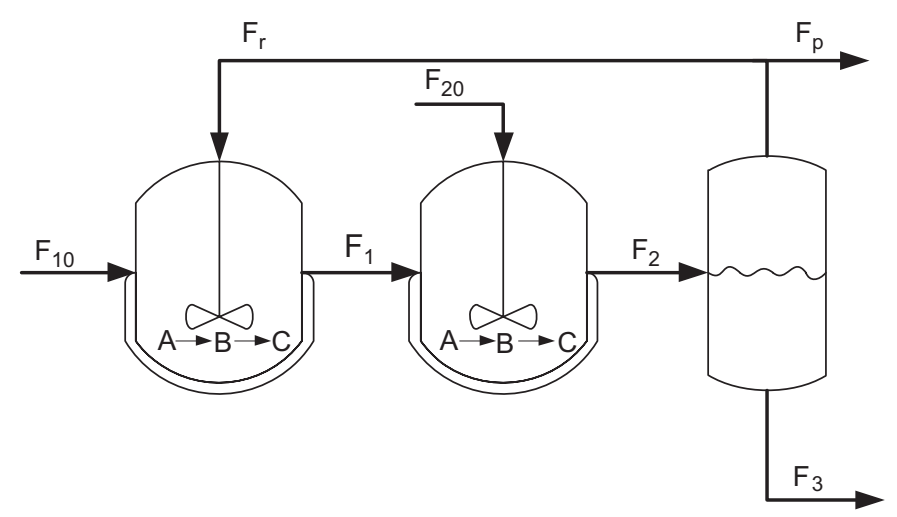

Fig. 6. Reactor-separator system with recycle.

desired product $B$ can then further react to form an undesired side-product $C$. The effluent of the first CSTR along with additional fresh feed $F_{20}$ makes up the inlet to the second CSTR. The reactions $A \rightarrow B$ and $B \rightarrow C$ (referred to as 1 and 2, respectively) take place in the two CSTRs in series before the effluent from CSTR 2 is fed to a flash tank. The overhead vapor from the flash tank is condensed and recycled to the first CSTR, and the bottom product stream is removed. A small portion of the overhead is purged before being recycled to the first CSTR. All the three vessels are assumed to have static holdup. The dynamic equations describing the behavior of the system, obtained through material and energy balances under standard modeling assumptions, are given as follows:

$$
\begin{aligned}
& \frac{\mathrm{d} x_{A 1}}{\mathrm{~d} t}=\frac{F_{10}}{V_{1}}\left(x_{A 10}-x_{A 1}\right)+\frac{F_{r}}{V_{1}}\left(x_{A r}-x_{A 1}\right)-k_{1} \mathrm{e}^{-E_{1} / R T_{1}} x_{A 1} \\
& \frac{\mathrm{d} x_{B 1}}{\mathrm{~d} t}=\frac{F_{10}}{V_{1}}\left(x_{B 10}-x_{B 1}\right)+\frac{F_{r}}{V_{1}}\left(x_{B r}-x_{B 1}\right) \\
& +k_{1} \mathrm{e}^{-E_{1} / R T_{1}} x_{A 1}-k_{2} \mathrm{e}^{-E_{2} / R T_{1}} x_{B 1} \\
& \frac{\mathrm{d} T_{1}}{\mathrm{~d} t}=\frac{F_{10}}{V_{1}}\left(T_{10}-T_{1}\right)+\frac{F_{r}}{V_{1}}\left(T_{3}-T_{1}\right) \\
& +\frac{-\Delta H_{1}}{C_{p}} k_{1} \mathrm{e}^{-E_{1} / R T_{1}} x_{A 1}+\frac{-\Delta H_{2}}{C_{p}} k_{2} \mathrm{e}^{-E_{2} / R T_{1}} x_{B 1} \\
& +\frac{Q_{1}}{\rho C_{p} V_{1}} \\
& \frac{\mathrm{d} x_{A 2}}{\mathrm{~d} t}=\frac{F_{1}}{V_{2}}\left(x_{A 1}-x_{A 2}\right)+\frac{F_{20}}{V_{2}}\left(x_{A 20}-x_{A 2}\right)-k_{1} \mathrm{e}^{-E_{1} / R T_{2} x_{A 2}} \\
& \frac{\mathrm{d} x_{B 2}}{\mathrm{~d} t}=\frac{F_{1}}{V_{2}}\left(x_{B 1}-x_{B 2}\right)+\frac{F_{20}}{V_{2}}\left(x_{B 20}-x_{B 2}\right) \\
& +k_{1} \mathrm{e}^{-E_{1} / R T_{2}} x_{A 2}-k_{2} \mathrm{e}^{-E_{2} / R T_{2}} x_{B 2} \\
& \frac{\mathrm{d} T_{2}}{\mathrm{~d} t}=\frac{F_{1}}{V_{2}}\left(T_{1}-T_{2}\right)+\frac{F_{20}}{V_{2}}\left(T_{20}-T_{2}\right) \\
& +\frac{-\Delta H_{1}}{C_{p}} k_{1} \mathrm{e}^{-E_{1} / R T_{2}} x_{A 2}+\frac{-\Delta H_{2}}{C_{p}} k_{2} \mathrm{e}^{-E_{2} / R T_{2}} x_{B 2} \\
& +\frac{Q_{2}}{\rho C_{p} V_{2}} \\
& \frac{\mathrm{d} x_{A 3}}{\mathrm{~d} t}=\frac{F_{2}}{V_{3}}\left(x_{A 2}-x_{A 3}\right)-\frac{F_{r}+F_{p}}{V_{3}}\left(x_{A r}-x_{A 3}\right) \\
& \frac{\mathrm{d} x_{B 3}}{\mathrm{~d} t}=\frac{F_{2}}{V_{3}}\left(x_{B 2}-x_{B 3}\right)-\frac{F_{r}+F_{p}}{V_{3}}\left(x_{B r}-x_{B 3}\right)
\end{aligned}
$$

Table 3

Process variables

\begin{tabular}{ll}
\hline$x_{A 1}, x_{A 2}, x_{A 3}$ & Mass fractions of $A$ in vessels $1-3$ \\
$x_{B 1}, x_{B 2}, x_{B 3}$ & Mass fractions of $B$ in vessels $1-3$ \\
$x_{C 1}, x_{C 2}, x_{C 3}$ & Mass fractions of $C$ in vessels $1-3$ \\
$x_{A r}, x_{B r}, x_{C r}$ & Mass fractions of $A-C$ in the recycle stream \\
$T_{1}, T_{2}, T_{3}$ & Temperatures in vessels $1-3$ \\
$T_{10}, T_{20}$ & Feed stream temperatures to vessels 1 and 2 \\
$F_{1}, F_{2}$ & Effluent flow rate from vessels 1 and 2 \\
$F_{10}, F_{20}$ & Steady-state feed stream flow rates to vessels 1 and 2 \\
$F_{r}, F_{p}$ & Flow rates of the recycle and purge streams \\
$V_{1}, V_{2}, V_{3}$ & Volumes of vessels $1-3$ \\
$E_{1}, E_{2}$ & Activation energy for reactions $(1)$ and (2) \\
$k_{1}, k_{2}$ & Pre-exponential values for reactions (1) and (2) \\
$\Delta H_{1}, \Delta H_{2}$ & Heats of reaction for reactions $(1)$ and (2) \\
$\alpha_{A}, \alpha_{B}, \alpha_{C}$ & Relative volatilities of $A-C$ \\
$Q_{1}, Q_{2}, Q_{3}$ & Heat inputs into vessels $1-3$ \\
$C_{p}, R, \rho$ & Heat capacity, gas constant and solution density \\
\hline
\end{tabular}

Table 4

Process parameters

\begin{tabular}{ll}
\hline$T_{10}$ & $300 \mathrm{~K}$ \\
$T_{20}$ & $300 \mathrm{~K}$ \\
$F_{10}$ & $5.04 \mathrm{~m}^{3} / \mathrm{h}$ \\
$F_{r}$ & $50.4 \mathrm{~m}^{3} / \mathrm{h}$ \\
$F_{p}$ & $5.04 \mathrm{~m}^{3} / \mathrm{h}$ \\
$V_{1}$ & $1.0 \mathrm{~m}^{3}$ \\
$V_{2}$ & $0.5 \mathrm{~m}^{3}$ \\
$V_{3}$ & $1.0 \mathrm{~m}^{3}$ \\
$E_{1}$ & $5 \times 10^{4} \mathrm{KJ} / \mathrm{kmol}$ \\
$E_{2}$ & $6 \times 10^{4} \mathrm{KJ} / \mathrm{kmol}$ \\
$k_{1}$ & $2.77 \times 10^{3} \mathrm{~s}^{-1}$ \\
$k_{2}$ & $2.5 \times 10^{3} \mathrm{~s}^{-1}$ \\
$\Delta H_{1}$ & $-6 \times 10^{4} \mathrm{KJ} / \mathrm{kmol}$ \\
$\Delta H_{2}$ & $-7 \times 10^{4} \mathrm{KJ} / \mathrm{kmol}$ \\
$\alpha_{A}$ & 3.5 \\
$\alpha_{B}$ & 1 \\
$\alpha_{C}$ & 0.5 \\
$C_{p}$ & $4.2 \mathrm{KJ} / \mathrm{kg} \mathrm{K}$ \\
$R$ & $8.314 \mathrm{KJ} / \mathrm{kmol} \mathrm{K}$ \\
$\rho$ & $1000 \mathrm{~kg} / \mathrm{m}^{3}$ \\
\hline
\end{tabular}

$\frac{\mathrm{d} T_{3}}{\mathrm{~d} t}=\frac{F_{2}}{V_{3}}\left(T_{2}-T_{3}\right)+\frac{Q_{3}}{\rho C_{p} V_{3}}$

where the definitions for the variables can be found in Table 3, with the parameter values given in Table 4 . The model of the flash tank separator operates under the assumption that the relative volatility for each of the species remains constant within the operating temperature range of the flash tank. This assumption allows calculating the mass fractions in the overhead based upon the mass fractions in the liquid portion of the vessel. It has also been assumed that there is a negligible amount of reaction taking place in the separator. The following algebraic equations model the composition of the overhead stream relative to the composition of the liquid holdup in the flash tank:

$$
\begin{aligned}
& x_{A r}=\frac{\alpha_{A} x_{A 3}}{\alpha_{A} x_{A 3}+\alpha_{B} x_{B 3}+\alpha_{C} x_{C 3}} \\
& x_{B r}=\frac{\alpha_{B} x_{B 3}}{\alpha_{A} x_{A 3}+\alpha_{B} x_{B 3}+\alpha_{C} x_{C 3}} \\
& x_{C r}=\frac{\alpha_{C} x_{C 3}}{\alpha_{A} x_{A 3}+\alpha_{B} x_{B 3}+\alpha_{C} x_{C 3}}
\end{aligned}
$$

Each of the tanks has an external heat input. The manipulated inputs to the system are the heat inputs to the three vessels, $Q_{1}, Q_{2}$ and $Q_{3}$, and the feed stream flow rate to vessel $2, F_{20}$.

System (17) was numerically simulated using a standard Euler integration method. The system was modeled with process noise. 
Table 5

Noise parameters

\begin{tabular}{lrlr}
\hline & $\sigma_{p}$ & $\phi$ & $\theta_{p}$ \\
\hline$x_{A 1}$ & 1 & 0.7 & 2 \\
$x_{B 1}$ & 1 & 0.7 & 2 \\
$T_{1}$ & 10 & 0.7 & 20 \\
$x_{A 2}$ & 1 & 0.7 & 2 \\
$x_{B 2}$ & 1 & 0.7 & 2 \\
$T_{2}$ & 10 & 0.7 & 20 \\
$x_{A 3}$ & 1 & 0.7 & 2 \\
$x_{B 3}$ & 1 & 0.7 & 2 \\
$T_{3}$ & 10 & 0.7 & 20 \\
\hline
\end{tabular}

Table 6

Steady-state values of manipulated inputs

\begin{tabular}{ll}
\hline Parameters & Values \\
\hline$Q_{1 s}$ & $12.6 \times 10^{5} \mathrm{KJ} / \mathrm{h}$ \\
$Q_{2 s}$ & $16.2 \times 10^{5} \mathrm{KJ} / \mathrm{h}$ \\
$Q_{3 s}$ & $12.6 \times 10^{5} \mathrm{KJ} / \mathrm{h}$ \\
$F_{20 s}$ & $5.04 \mathrm{~m}^{3} / \mathrm{h}$ \\
\hline
\end{tabular}

Process noise was added to the right-hand side of each equation in the system of ODEs found in Eq. (17) and generated as autocorrelated noise of the form $w_{k}=\phi w_{k-1}+\xi_{k}$ where $k=0,1, \ldots$ is the discrete time step of $0.001 \mathrm{~h}, \xi_{k}$ is generated by a normally distributed random variable with standard deviation $\sigma_{p}$ and $\phi$ is the autocorrelation factor and $w_{k}$ is bounded by $\theta_{p}$, that is $\left|w_{k}\right| \leqslant \theta_{p}$. Table 5 contains the parameters used in generating the process noise.

We assume that the measurements of temperatures $T_{1}, T_{2}$ and $T_{3}$ are available continuously, and the measurements of mass fractions $x_{A 1}, x_{B 1}, x_{A 2}, x_{B 2}, x_{A 3}$ and $x_{B 3}$ are available asynchronously at time instants $\left\{t_{k} \geqslant 0\right\}$ and are subject to time-varying measurement delay. We also assume that there exists a lower bound $T_{\min }=0.2 \mathrm{~h}$ on the time interval between two consecutive measurements of the mass fractions. The same method used in the example in Section 4 is used in the present example to generate the time sequence $\left\{t_{k} \geqslant 0\right\}$. For each set of steady-state inputs $Q_{1 s}, Q_{2 s}, Q_{3 s}$ and $F_{20 s}$ corresponding to a different operating condition, system (17) has one stable steadystate $x_{S}^{\mathrm{T}}$. The control objective is to steer the system from the initial state

$x(0)^{\mathrm{T}}=[0.890,0.110,388.7,0.886,0.113,386.3,0.748,0.251,390.6]$

to the steady-state

$x_{s}^{\mathrm{T}}=[0.383,0.581,447.8,0.391,0.572,444.6,0.172,0.748,449.6]$

corresponding to the operating condition presented in Table 6 .

The process model (17) belongs to the class of nonlinear systems

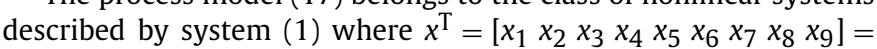
$\left[x_{A 1}-x_{A 1 s} x_{B 1}-x_{B 1 s} T_{1}-T_{1 s} x_{A 2}-x_{A 2 s} x_{B 2}-x_{B 2 s} T_{2}-T_{2 s} x_{A 3}-\right.$ $\left.x_{A 3 s} x_{B 3}-x_{B 3 s} T_{3}-T_{3 s}\right]$ is the state, $u_{s}^{\mathrm{T}}=\left[\begin{array}{lll}u_{s 1} & u_{s 2} & u_{s 3}\end{array}\right]=\left[Q_{1}-Q_{1 s} Q_{2}-\right.$ $Q_{2 s} Q_{3}-Q_{3 s}$ ] and $u_{d}=F_{20}-F_{20 s}$ are the manipulated inputs, $w=$ $w_{k}$ is a time varying bounded noise, $y_{s}^{\mathrm{T}}=\left[\begin{array}{lll}y_{s 1} & y_{s 2} & y_{s 3}\end{array}\right]=\left[\begin{array}{lll}x_{3} & x_{6} & x_{9}\end{array}\right]$ is obtained from the continuous temperature measurements and $y_{d}^{\mathrm{T}}=\left[\begin{array}{llllll}x_{1} & x_{2} & x_{4} & x_{5} & x_{7} & x_{8}\end{array}\right]$ is obtained from the sampled asynchronous, delayed mass fraction measurements.

The performance index used in the present example is defined by a positive definite function $L\left(x, u_{s}, u_{d}\right)$ as in Eq. (16) with $Q_{c}$ being
Table 7

Control parameters for steady-state $x_{s}$

\begin{tabular}{lr}
\hline Parameters & Values \\
\hline$K_{1}$ & -5000 \\
$K_{2}$ & -5000 \\
$K_{3}$ & -5000 \\
$T_{i}$ & $5 \mathrm{~h}$ \\
\hline
\end{tabular}

the following weight matrix

$Q_{c}=\operatorname{diag}^{6}\left(10^{4} 10^{4} 110^{4} 10^{4} 110^{4} 10^{4} 1\right)$

The values of the weights in $Q_{c}$ have been chosen to account for the different range of numerical values for each state.

\subsection{Lower-tier controller design}

Based on the continuous temperature measurements (i.e., $y_{S}(t)$ ), three output feedback controllers (lower-tier controllers) are first designed to stabilize system (17) from the initial state $x(0)$ to the steady-state $x_{S}$ using only the heat inputs $u_{S}$ as the manipulated inputs. In particular, three PI controllers are used as the lower-tier controllers of the following form:

$u_{s j}(t)=K_{j}\left(y_{s j}(t)+\frac{1}{T_{i j}} \int_{0}^{t} y_{s j}(\tau) \mathrm{d} \tau\right)$

where $j=1,2,3, K_{j}$ are the proportional gains and $T_{i j}$ are the integral time constants.

As in the first example, to compute the parameters of the controllers, the linearized model $\dot{x}=A x+B u_{s}$ of system (17) around the equilibrium point is obtained. The parameters of the lower-tier controllers are presented in Table 7. These values guarantee that the origin of the linear system

$\dot{x}=A_{C} x$

where

$A_{C}=A+B\left[\begin{array}{ccc}K_{1} & 0 & 0 \\ 0 & K_{2} & 0 \\ 0 & 0 & K_{3}\end{array}\right]\left[\begin{array}{lllllllll}0 & 0 & 1 & 0 & 0 & 0 & 0 & 0 & 0 \\ 0 & 0 & 0 & 0 & 0 & 1 & 0 & 0 & 0 \\ 0 & 0 & 0 & 0 & 0 & 0 & 0 & 0 & 1\end{array}\right]$

is asymptotically stable with the real parts of the eigenvalues of $A_{c}$ being $\lambda_{\operatorname{Re}}=[-249-73.9-73.9-2.07-2.07-131-108-24.0-$ $56.4]^{\mathrm{T}}$. Solving the algebraic Lyapunov equation $A_{c}^{\mathrm{T}} P+P A_{c}+Q_{c}=0$, a quadratic Lyapunov function $V(x)=x^{\mathrm{T}} P x$ is obtained. The Lyapunov function is used to design the upper-tier LMPC controller. The state and input trajectories of system (17) under the PI control law (19) are shown in Figs. 7 and 8. From Fig. 7, we see that the PI control law (19) drives the temperatures and mass fractions in the three vessels close to the equilibrium point in about $0.7 \mathrm{~h}$. For a simulation length of $t_{f}=1 \mathrm{~h}$, the performance cost associated with the resulting closed-loop trajectories is $2.105 \times 10^{5}$.

\subsection{Two-tier control architecture design}

We design next the upper-tier LMPC controller. The feed flow rate to vessel $2, u_{d}=F_{20}-F_{20}$, is the manipulated input for the LMPC, which is bounded by $1 \leqslant F_{20} \leqslant 9 \mathrm{~m}^{3} / \mathrm{h}$. The sampling time of the LMPC is chosen to be $\Delta=0.025 \mathrm{~h}$; the prediction horizon is chosen to be $\tau_{f}=8 \Delta=0.2 \mathrm{~h}$ so that the prediction captures most of the dynamic evolution of the process; the quadratic control Lyapunov function $V(x)$ obtained in Section 5.2 is used in the design of the contractive

${ }^{6}$ We use $\operatorname{diag}(V)$ to denote a matrix with its diagonal elements being the elements of vector $V$ and all the other elements being zeros. 

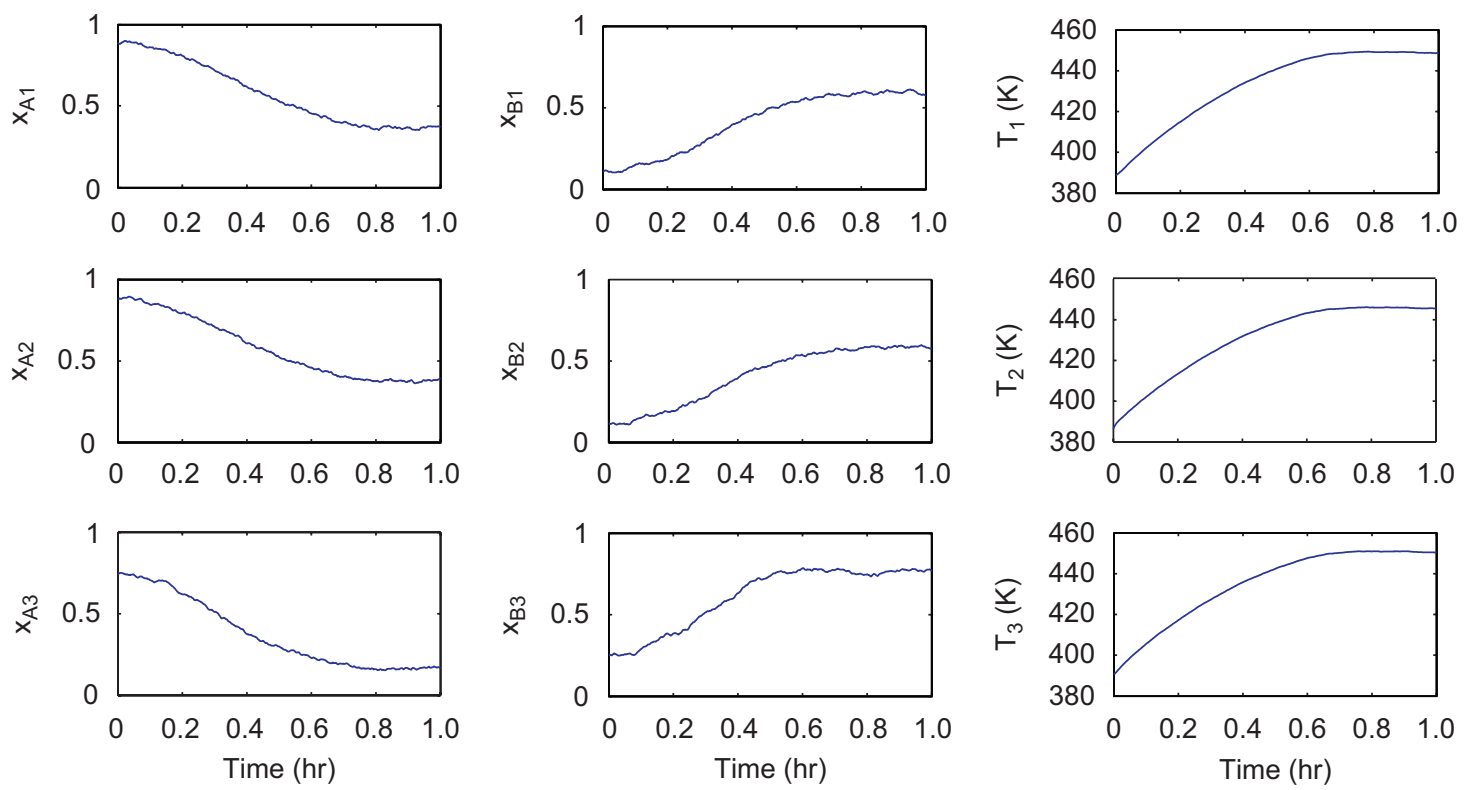

Fig. 7. State trajectories of system (17) under lower-tier control law (19).
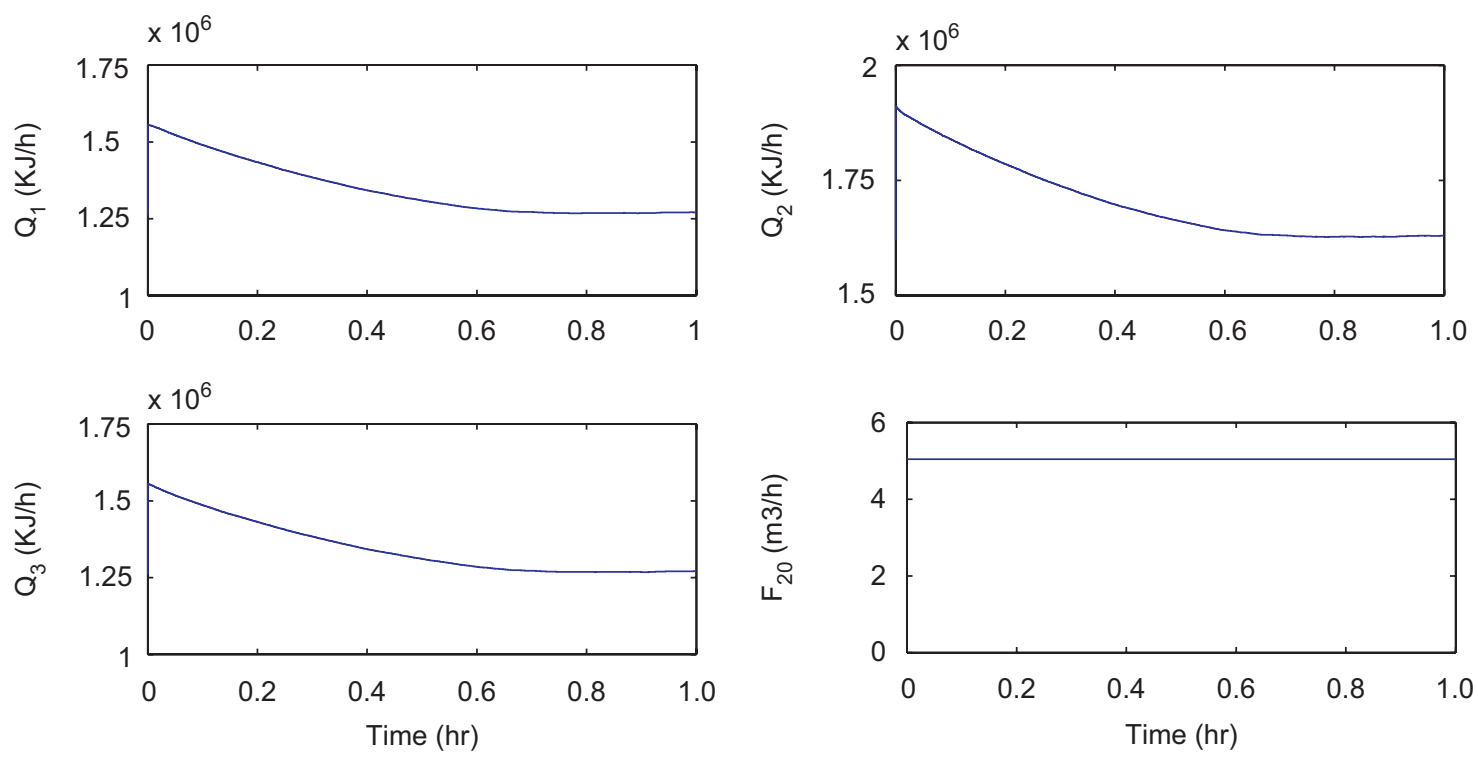

Fig. 8. Input trajectories of system (17) under lower-tier PI control $(19)\left(u_{d}(t) \equiv 0\right)$.

constraint (3h). For the simulations carried out in this subsection, we set the prediction horizon $\tau_{f}$ to be equal to the minimum time interval between two consecutive $y_{d}$ measurements, $T_{\min }$, and the delay associated with each measurement to be $d_{k}=D=0.2 \mathrm{~h}$ for all $k$ which also corresponds to the worst-case effect of measurement delay.

The two-tier control architecture (4) is implemented as discussed in Section 3. The mass fraction measurement sequence $\left\{t_{k} \geqslant 0\right\}$ (generated with $W=1$ ) and the delay size sequence $\left\{d_{k} \geqslant 0\right\}$ with a simulation length $0.75 \mathrm{~h}$ are as follows:

$$
\begin{aligned}
& \left\{t_{k \geqslant 0}\right\}=\{0,0.248,0.495,0.868,1.000\} \\
& \left\{d_{k} \geqslant 0\right\}=\{0,0.200,0.200,0.200,0.200\}
\end{aligned}
$$

The state and input trajectories of system (17) under the proposed two-tier control architecture are shown in Figs. 9 and 10. Fig. 9 shows that the two-tier control architecture (4) drives the temperatures and the mass fractions in the closed-loop system close to the equilibrium point in about $0.25 \mathrm{~h}$. This implies that the resulting closed-loop system response is faster relative to the speed of the closed-loop response under the lower-tier PI controllers. For the same simulation length of $t_{f}=1 \mathrm{~h}$, the performance cost associated with the resulting closed-loop trajectories is $8.658 \times 10^{4}$ which is much smaller than that of the closed-loop system under the lower-tier PI control system $\left(2.105 \times 10^{5}\right)$.

\subsection{Performance comparison}

We carried out a set of simulations to compare the proposed two-tier control architecture with the lower-tier PI control system with the same parameters from a performance index point of view. Table 8 presents the total cost computed for 10 different closed-loop 

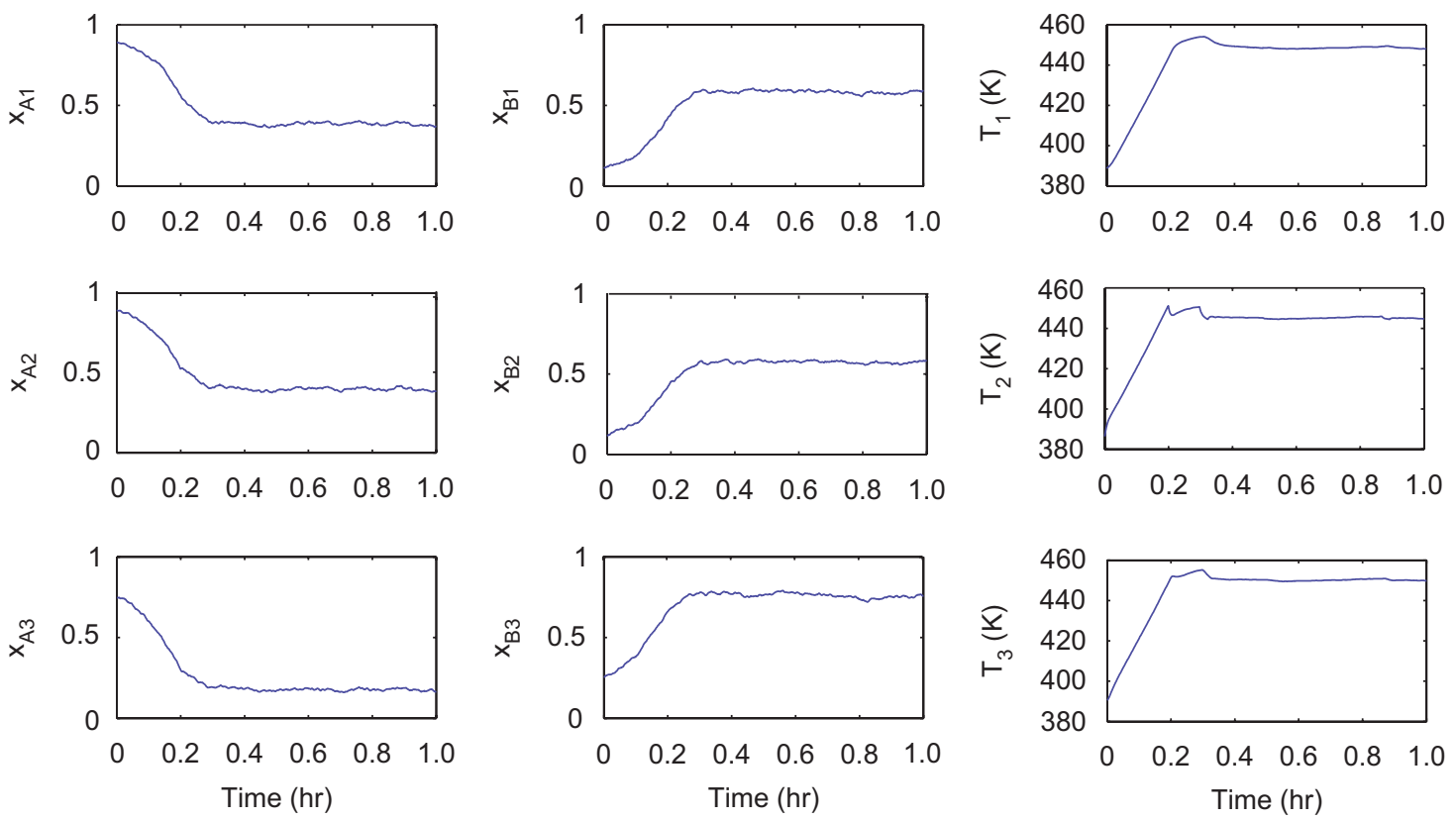

Fig. 9. State trajectories of system (17) under the proposed two-tier control architecture.
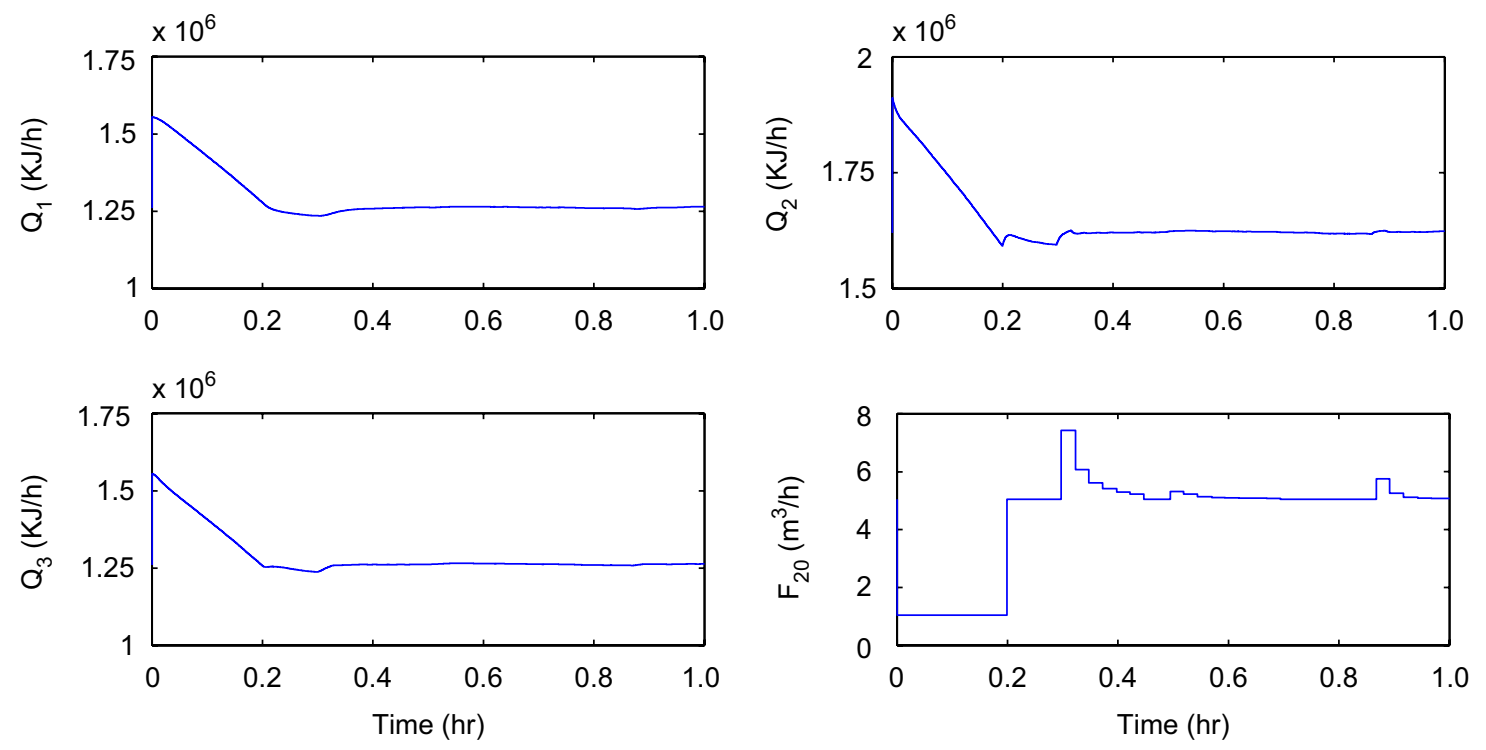

Fig. 10. Input trajectories of system (17) under the proposed two-tier control architecture.

Table 8

Total performance cost along the closed-loop system trajectories

\begin{tabular}{rll}
\hline Sim. & Two-tier & PI control \\
\hline 1 & $1.006 \times 10^{4}$ & $2.148 \times 10^{4}$ \\
2 & $2.046 \times 10^{4}$ & $3.123 \times 10^{4}$ \\
3 & $3.621 \times 10^{4}$ & $6.310 \times 10^{4}$ \\
4 & $1.148 \times 10^{4}$ & $4.440 \times 10^{4}$ \\
5 & $3.103 \times 10^{4}$ & $6.052 \times 10^{4}$ \\
6 & $7.141 \times 10^{4}$ & $1.631 \times 10^{5}$ \\
7 & $1.389 \times 10^{4}$ & $6.961 \times 10^{4}$ \\
8 & $1.928 \times 10^{4}$ & $2.770 \times 10^{4}$ \\
9 & $1.872 \times 10^{4}$ & $8.538 \times 10^{4}$ \\
10 & $1.417 \times 10^{4}$ & $7.260 \times 10^{4}$ \\
\hline
\end{tabular}

simulations under the proposed two-tier control architecture and the lower-tier PI control system. To carry out this comparison, we have computed the total cost of each simulation based on the integral of the performance index defined by $L\left(x, u_{s}, u_{d}\right)$ with different operating conditions. The length of each simulation is $t_{f}=0.75 \mathrm{~h}$. For this set of simulations $W$ is chosen to be 1 . For each pair of simulations (one for each control scheme) a different initial state inside the stability region, a different noise trajectory and a different random mass fraction measurement sequence with random delay size sequence are generated by the methods described in Section 5.1. As can be seen in Table 8, the proposed two-tier control architecture has a cost lower than the corresponding total cost under the lowertier PI control system in all the simulations. 

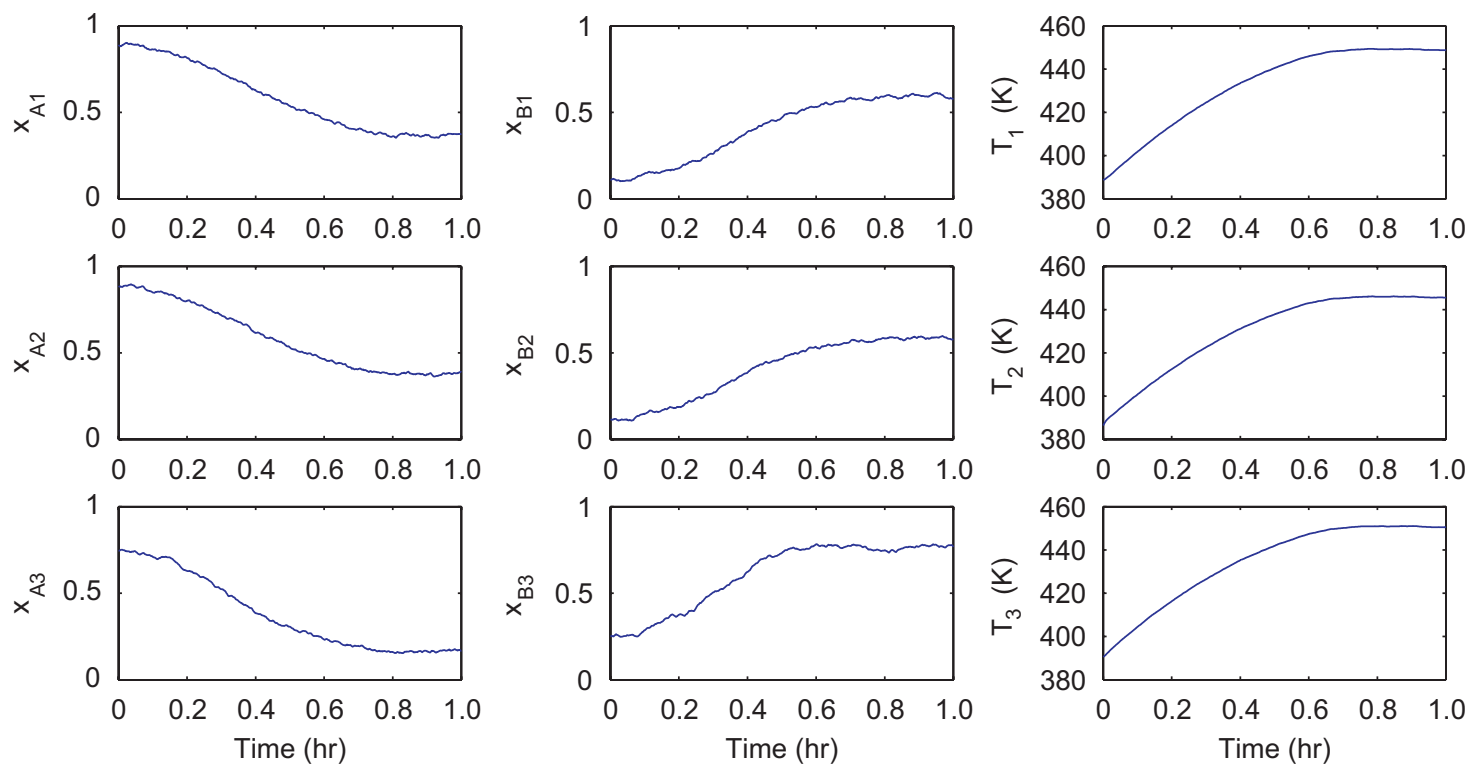

Fig. 11. State trajectories of system (17) subject to input constraints under the lower-tier control law (19).
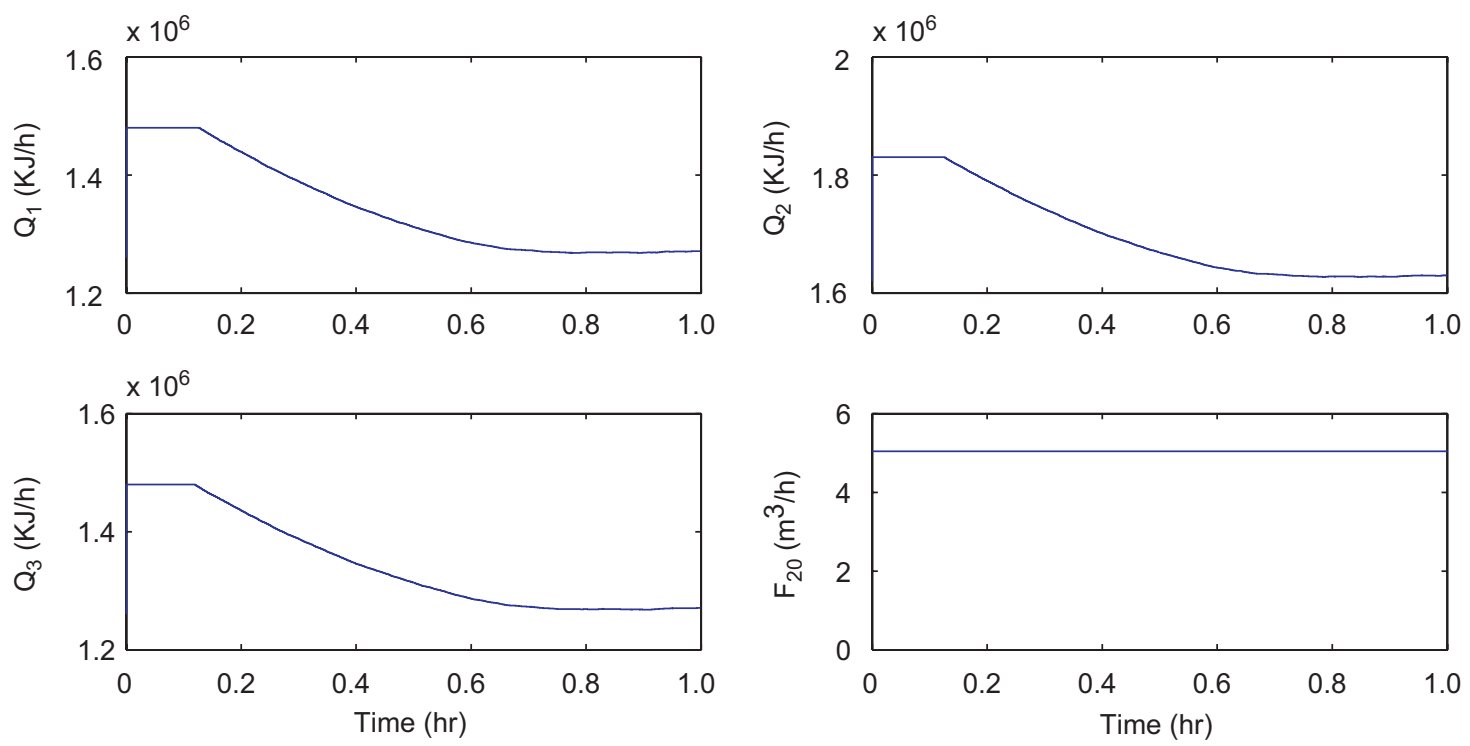

Fig. 12. Input trajectories of system (17) subject to input constraints under the lower-tier control law (19).

\subsection{Effect of input constraints}

In this section, we study the effect of input constraints on the performance of the closed-loop system under the proposed twotier control architecture. Specifically, in the simulations carried out in this subsection, we take into account input constraints in the lower-tier controller manipulated inputs $u_{s}$, namely $\left|Q_{1}\right| \leqslant 1.48 \times 10^{5}$, $\left|Q_{2}\right| \leqslant 1.83 \times 10^{5}$ and $\left|Q_{3}\right| \leqslant 1.48 \times 10^{5} \mathrm{KJ} / \mathrm{h}$. The same simulation settings (initial condition, target state, lower-tier controller design, upper-tier controller design, mass fraction measurement sequence and delay size sequence) as in Sections 5.1-5.3 are used in the simulations carried out in this subsection.

The state and input trajectories under the lower-tier PI controllers (19) are shown in Figs. 11 and 12. From Fig. 11, we see that the PI controllers stabilize the system at the target steady-state in about $0.8 \mathrm{~h}$ which is a little slower than the corresponding closed-loop response without input constraints (in such a case the closed-loop system is stabilized in about $0.7 \mathrm{~h}$ ). From Fig. 12, we see that the three heat inputs $Q_{1}, Q_{2}$ and $Q_{3}$ operate at their maximum allowable values for about $0.15 \mathrm{~h}$. The corresponding accumulated performance cost is $2.180 \times 10^{5}$.

The state and input trajectories under the two-tier control architecture (4) with the same upper-tier controller design is shown in Figs. 13 and 14. Fig. 13 shows that the two-tier control architecture (4) drives the temperatures and the mass fractions of the closedloop system close to the equilibrium point in about $0.3 \mathrm{~h}$ which is a little slower than the closed-loop system response without input constraints (in this case the closed-loop system stabilizes in about $0.25 \mathrm{~h}$ ). From Fig. 14, we see that the heat inputs $Q_{1}, Q_{2}$ and $Q_{3}$ also operate at their maximum allowable values for about $0.15 \mathrm{~h}$. The corresponding accumulated performance cost is $9.443 \times 10^{4}$ which is much lesser than the cost obtained under the lower-tier control 

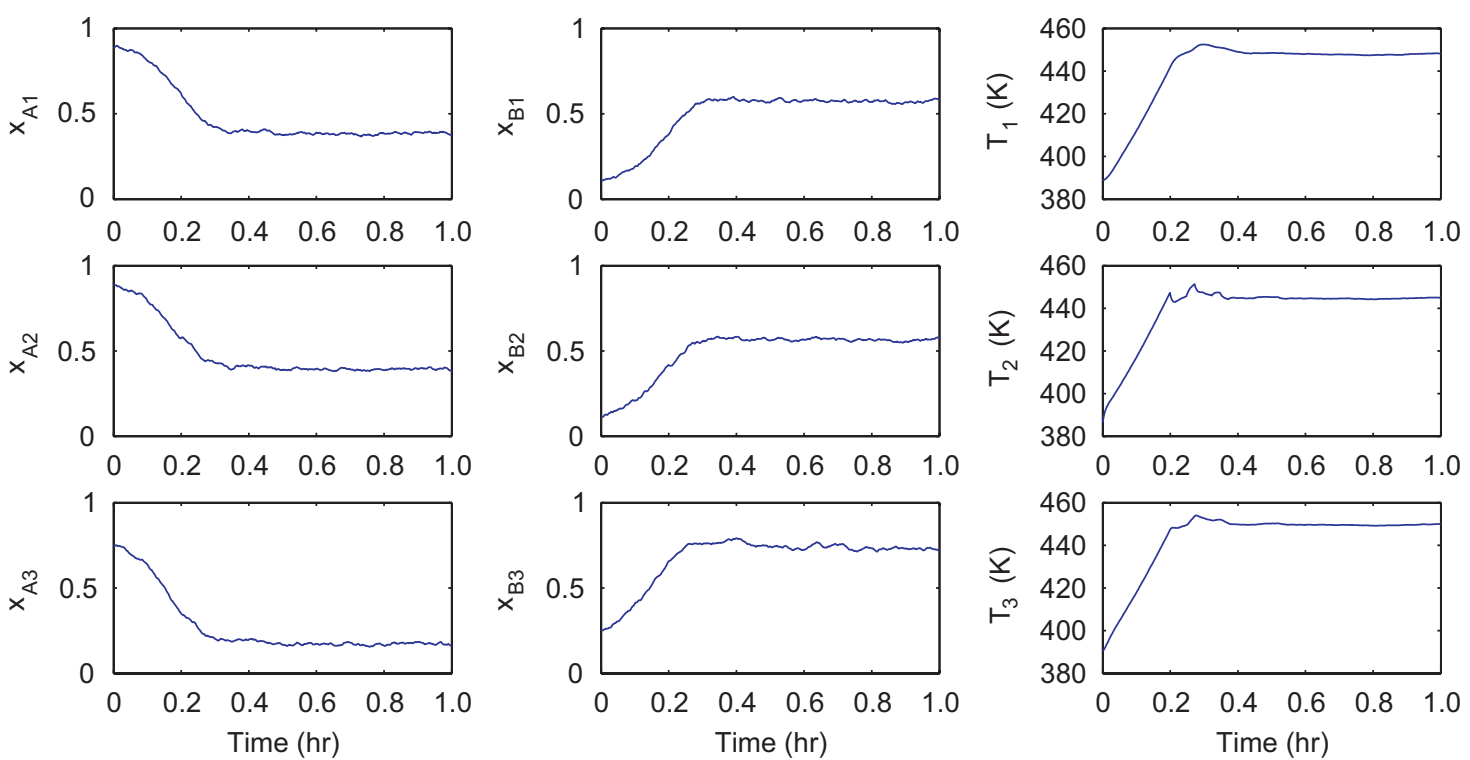

Fig. 13. State trajectories of system (17) subject to input constraints under the proposed two-tier control architecture.
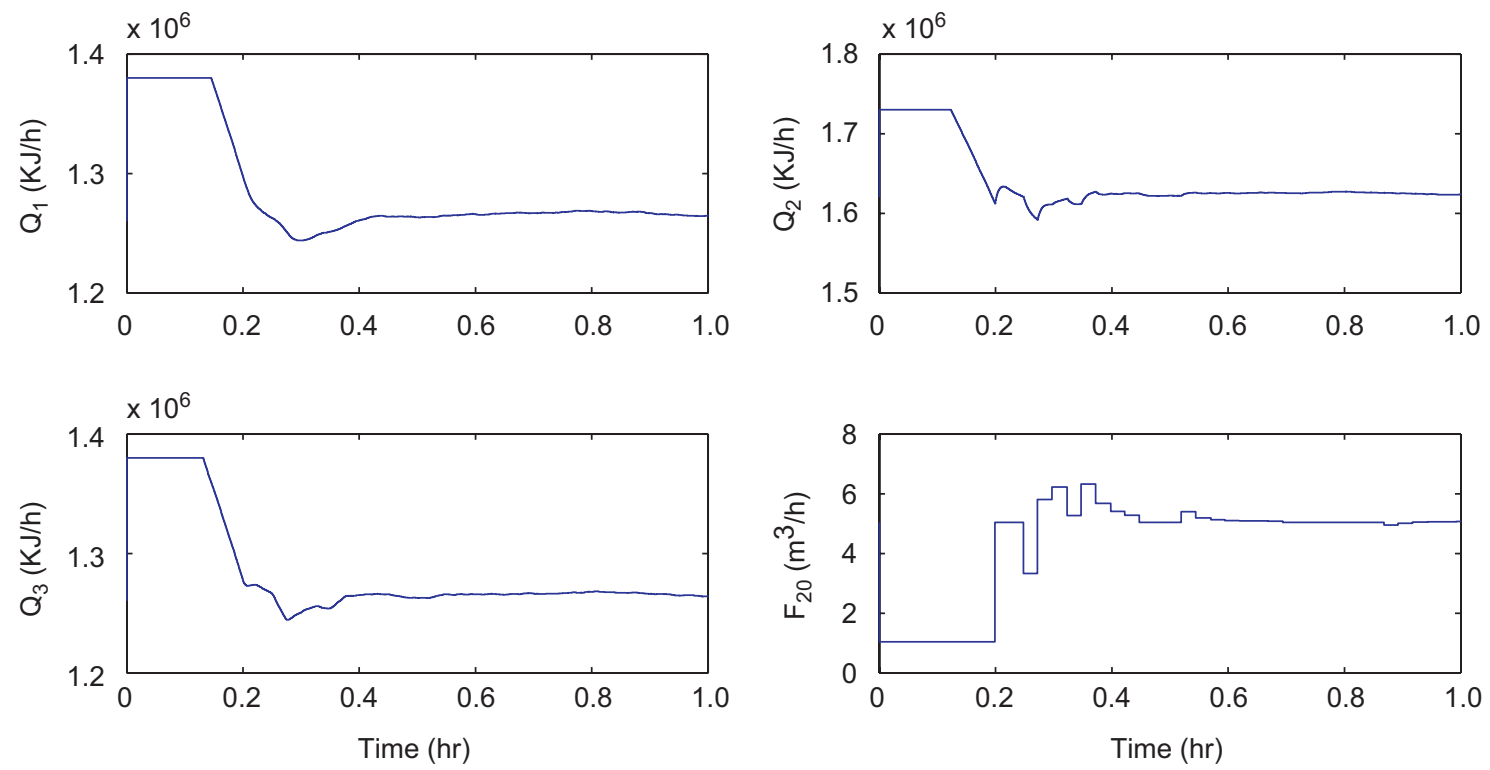

Fig. 14. Input trajectories of system (17) subject to input constraints under the proposed two-tier control architecture.

system $\left(2.180 \times 10^{5}\right)$. From this set of simulations, we see that the proposed two-tier control architecture maintains the property of improving the performance of the closed-loop system when input constraints are present. It is also important to note that advanced anti-windup schemes could be used in conjunction with the lowertier PI controllers to mitigate the effect of integrator wind-up and improve the closed-loop system performance; however, the basic conclusion of this part of the study would not change.

Remark 19. In some applications, when input constraints are present, the stability of the closed-loop system under the lower-tier controller may be lost because of saturation of the control inputs. To avoid losing stability, the lower-tier controller in the two-tier control architecture can be detuned to primarily take care of the closed-loop system stability by sacrificing closed-loop performance.
Thus, when input constraints are present, the lower-tier controller can be potentially detuned to satisfy the input constraints (or saturate for less time) and the upper-tier controller can be used to recover the loss of closed-loop performance.

\section{Conclusions}

Traditionally, process control systems utilize dedicated, point-topoint wired communication links using a small number of sensors and actuators to regulate appropriate process variables at desired values. While this paradigm to process control has been successful, chemical plant operation could substantially benefit from an efficient integration of the existing, point-to-point control networks (wired connections from each actuator/sensor to the control system using dedicated local area networks) with additional networked (wired or 
wireless) actuator/sensor devices. However, available control methods cannot be used for the design of process control systems which utilize networked sensors and actuators because they do not deal directly with data losses due to field interference and time-delays due to network traffic at the controller design level. Motivated by these technological advances and the lack of methods to design control systems that utilize hybrid communication networks, we presented in this work a two-tier control architecture for process control problems that involve nonlinear processes and heterogeneous measurements consisting of continuous measurements and asynchronous, delayed measurements. The proposed architecture consists of: (a) a lower-tier control system, which relies on point-to-point communication and continuous measurements, to stabilize the closed-loop system, and (b) an upper-tier networked control system, designed using Lyapunov-based model predictive control theory, that profits from both the continuous and the asynchronous, delayed measurements as well as from additional networked control actuators to improve the closed-loop system performance. The applicability and effectiveness of the proposed control method was demonstrated using two chemical process examples and constraints on the achievable closed-loop system performance were evaluated.

\section{Acknowledgments}

Financial support from NSF, CBET-0529295 and MEC, DPI200766718-C04-01 is gratefully acknowledged.

\section{References}

Camponogara, E., Jia, D., Krogh, B.H., Talukdar, S., 2002. Distributed model predictive control. IEEE Control Systems Magazine 22, 44-52.

Christofides, P.D., El-Farra, N.H., 2005. Control of Nonlinear and Hybrid Process Systems: Designs for Uncertainty, Constraints and Time-Delays. Springer, Berlin, Germany.

Christofides, P.D., Davis, J.F., El-Farra, N.H., Clark, D., Harris, K.R.D., Gipson, J.N., 2007. Smart plant operations: vision, progress and challenges. A.I.Ch.E. Journal 53, 2734-2741.

Davis, J.F., 2007. Report from NSF workshop on cyberinfrastructure in chemical and biological systems: impact and directions (see (http://www.oit.ucla.edu/nsfci/ NSFCIFullReport.pdf $>$ for the pdf file of this report).

Gao, H., Chen, T., Lam, J., 2008. A new delay system approach to network-based control. Automatica 44, 39-52.

Ghantasala, S., El-Farra, N.H., 2008. Robust diagnosis and fault-tolerant control of distributed processes over communication networks. International Journal of Adaptive Control and Signal Processing, in press.
Jeong, S.C., Park, P., 2005. Constrained MPC algorithm for uncertain time-varying systems with state-delay. IEEE Transactions on Automatic Control 50, 257-263. Khalil, H.K., 1996. Nonlinear Systems. second ed. Prentice-Hall, NY.

Lian, F.-L., Moyne, J., Tilbury, D., 2003. Modelling and optimal controller design of networked control systems with multiple delays. International Journal of Control 76, 591-606.

Liu, G.-P., Xia, Y., Chen, J., Rees, D., Hu, W., 2007. Networked predictive control of systems with random network delays in both forward and feedback channels. IEEE Transactions on Industrial Electronics 54, 1282-1297.

Liu, J., Muñoz de la Peña, D., Ohran, B., Christofides, P.D., Davis, J.F., submitted for publication. A two-tier control architecture for nonlinear process systems with continuous/asynchronous feedback. International Journal of Control.

Liu, J., Muñoz de la Peña, D., Christofides, P.D., Davis, J.F., in press. Lyapunovbased model predictive control of nonlinear systems subject to time-varying measurement delays. International Journal of Adaptive Control and Signal Processing.

Mhaskar, P., El-Farra, N.H., Christofides, P.D., 2005. Predictive control of switched nonlinear systems with scheduled mode transitions. IEEE Transactions on Automatic Control 50, 1670-1680.

Mhaskar, P., El-Farra, N.H., Christofides, P.D., 2006. Stabilization of nonlinear systems with state and control constraints using Lyapunov-based predictive control. Systems \& Control Letters 55, 650-659.

Montestruque, L.A., Antsaklis, P.J., 2004. Stability and persistent disturbance attenuation properties for a class of networked control systems: switched system approach. IEEE Transactions on Automatic Control 49 (9), 1562-1572.

Muñoz de la Peña, D., Christofides, P.D., 2008. Lyapunov-based model predictive control of nonlinear systems subject to data losses. IEEE Transactions on Automatic Control, in press.

Nešić, D., Teel, A., Kokotovic, P., 1999. Sufficient conditions for stabilization of sampled-data nonlinear systems via discrete time approximations. Systems and Control Letters 38, 259-270.

Neumann, P., 2007. Communication in industrial automation-what is going on? Control Engineering Practice 15, 1332-1347.

Rawlings, J.B., Stewart, B.T., 2007. Coordinating multiple optimization-based controllers: New opportunities and challenges. In: Proceedings of 8th IFAC Symposium on Dynamics and Control of Process, vol. 1, Cancun, Mexico, pp. 19-28.

Sun, Y., El-Farra, N.H., 2008. Quasi-decentralized model-based networked control of process systems. Computers \& Chemical Engineering 32, 2016-2115.

Tabuada, P., Wang, X., 2006. Preliminary results on state-triggered scheduling of stabilizing control tasks. In: Proceedings of IEEE Conference on Decision and Control.pp. 282-287.

Wang, Y.M.L., Chu, T., Hao, F., 2005. Stabilization of networked control systems with data packet dropout and transmission delays: continuous-time case. European Journal of Control 11, 40-55.

Witrant, E., Georges, D., Canudas-de-Wit, C., Alamir, M., 2007. On the use of state predictors in networked control system. Applications of Time Delay Systems, Lecture Notes in Control and Information Sciences, vol. 352. pp. 17-35.

Ydstie, E.B., 2002. New vistas for process control: integrating physics and communication networks. A.I.Ch.E. Journal 48, 422-426.

Zhang, L., Shi, Y., Chen, T., Huang, B., 2005. A new method for stabilization of networked control systems with random delays. IEEE Transactions on Automatic Control 50, 1177-1181. 